\title{
A CHARACTERIZATION OF VIRTUALLY EMBEDDED SUBSURFACES IN 3-MANIFOLDS
}

\author{
YI LIU
}

\begin{abstract}
The paper introduces the spirality character of the almost fiber part for a closed essentially immersed subsurface of a closed orientable aspherical 3-manifold, which generalizes an invariant due to Rubinstein and Wang. The subsurface is virtually embedded if and only if the almost fiber part is aspiral, and in this case, the subsurface is virtually a leaf of a taut foliation. Besides other consequences, examples are exhibited that non-geometric 3-manifolds with no Seifert fibered pieces may contain essentially immersed but not virtually embedded closed subsurfaces.
\end{abstract}

\section{INTRODUCTION}

In this paper, we study virtually essentially embedded closed subsurfaces of closed orientable 3-manifolds. We always assume a closed surface or 3-manifold to be connected, but a bounded surface or 3-manifold may be disconnected. A closed locally flatly immersed subsurface $S$ of a 3 -manifold $M$ is said to be essentially immersed, if $S$ is not a sphere and no homotopically nontrivial loop in $S$ is contractible in $M$. A (homotopy) virtual property of $S$ is a property which holds for some lift of $S$ in some finite cover of $M$ up to homotopy.

While all essentially immersed subsurfaces of geometric 3-manifolds are known to be virtually embedded, which is a consequence of Agol's proof of the Virtual Haken Conjecture in the hyperbolic case [Ag2] and a simple fact in the non-hyperbolic case, cf. [NW], many essentially immersed subsurfaces of non-geometric 3-manifolds do not to have this property. The first such example was discovered by Rubinstein and Wang [RW]. In [PW3], Przytycki and Wise have shown that a closed essentially immersed subsurface of a 3-manifold is virtually embedded if and only if it is $\pi_{1^{-}}$ separable.

From a topological point of view, we will characterize virtually essentially embedded subsurfaces of 3-manifolds in terms of the spirality of the almost fiber part. Canonically induced from the JSJ decomposition, a closed essentially immersed subsurface $S$ of a closed orientable aspherical 3 -manifold $M$ can be decomposed into the JSJ subsurfaces along JSJ curves. The almost fiber part $\Phi(S)$ of the surface $S$ is thus defined to be the union of all the horizontal or geometrically infinite JSJ subsurfaces, carried by Seifert fibered or hyperbolic pieces of $M$ respectively, glued up along shared JSJ curves. Denote by $\mathbb{Q}^{\times}$the multiplicative abelian group of nonzero rational numbers. We will introduce the spirality character of the almost

Date: March 27, 2015.

2010 Mathematics Subject Classification. Primary 57M05.

Key words and phrases. spirality character, virtually embedded subsurface, suspension flow.

Partially supported by NSF grant DMS-1308836. 
fiber part $\Phi(S)$,

$$
s: H_{1}(\Phi(S) ; \mathbb{Z}) \rightarrow \mathbb{Q}^{\times},
$$

which is a natural invariant homomorphism. We say that $\Phi(S)$ is aspiral if the spirality character only takes the values \pm 1 . When $M$ is a graph manifold and $S$ is orientable and horizontally immersed, the spirality character coincides with the invariant $s$ introduced by Rubinstein-Wang [RW].

In the case that $S$ is virtually essentially embedded, it is also interesting to ask whether $S$ virtually fits into a taut foliation. Recall that a codimension-1 foliation of a 3-manifold is said to be taut if for every leaf of the foliation there exists an immersed loop passing through the leaf and transverse to all leaves of the foliation. When $S$ is orientable, the question is equivalent to whether $S$ virtually minimizes the Thurston norm, cf. [Th2, Ga].

The main result of this paper is the following:

Theorem 1.1. Let $M$ be an orientable closed aspherical 3-manifold, and $S$ be a closed essentially immersed subsurface. Then following statements are equivalent:

(1) $S$ is aspiral in the almost fiber part;

(2) $S$ is virtually essentially embedded;

(3) $S$ is virtually a leaf of a taut foliation.

Theorem 1.1 implies, at least in principle, a solution to the decision problem for (closed) surface subgroup separability in closed 3-manifold groups. For example, take the input a finite presentation of a closed 3-manifold group and a finite generating set of a surface subgroup:

Corollary 1.2. There exists an algorithm to decide whether or not a surface subgroup in a closed 3-manifold group is separable.

Although the algorithm asserted by Corollary 1.2 is not practical for implementation, the complexity of computation lies primarily in recognizing the almost fiber part and understanding how the JSJ subsurfaces serve as virtual fibers. Once this piece of information is provided, the spirality character can be easily calculated, (Formula 4.5). Furthermore, the situation can be significantly improved when the subsurface is immersed locally in a geometrically nice position. Especially, if the subsurface is an almost fiber transverse to a canonical suspension flow of a fibered cone, there is a formula for the spirality character generalizing the work of Rubinstein-Wang most directly, (Formula 7.2).

Let $\theta: F \rightarrow F$ be an orientation-preserving homeomorphism of an oriented closed surface $F$ of negative Euler characteristic. Denote by $M_{\theta}$ the mapping torus, which canonically fibers over the circle with monodromy $\theta$. Inspired by the works [RW, CLR1, CLR2], we investigate immersed subsurfaces $S$ transverse to either the Seifert fibration or the pseudo-Anosov suspension flow in the JSJ pieces.

The following Corollary 1.3 provides a practical criterion for aspirality without the necessity to understand the almost fiber part. See Subsection 2.2 for further terminology about surface automorphisms.

Corollary 1.3. Let $S$ be an oriented closed essentially immersed subsurface of $M_{\theta}$. Suppose all fractional Dehn twist coefficients of $\theta$ vanish. If $S$ can be homotoped to be transverse to the JSJ tori and to the Nielsen-Thurston suspension flow supported in the JSJ pieces, then $S$ is virtually embedded. 
On the other hand, we are able to produce essentially immersed but not virtually embedded closed subsurfaces, under the circumstances complementary to Corollary 1.3:

Corollary 1.4. If $\theta$ has a nontrivial fractional Dehn twist coefficient, then $M_{\theta}$ contains a closed essentially immersed subsurface that is not virtually embedded.

In particular, $\pi_{1}\left(M_{\theta}\right)$ cannot be LERF or even surface subgroup separable under the assumption of Corollary 1.4. Take the double of a pseudo-Anosov map of a bounded surface and compose with a nontrivial Dehn twist along a doubling curve. Since the surface automorphism has a nontrivial fractional Dehn twist coefficient but does not have Seifert fibered pieces in the mapping torus, we find a counterexample to disprove [AFW1, Conjecture 9.1]. Another consequence of Corollary 1.4, probably well known to experts, is that virtually fibered non-geometric graph manifolds all fail to have surface subgroup separability of the fundamental group. In fact, one may also remove the virtually fibered condition by a slightly more careful construction - compare [Ne, NW, RW]. Suggested by these examples, we are inclined to propose the following:

Conjecture 1.5. Every non-geometric closed aspherical 3-manifold contains a closed essentially immersed subsurface which is not virtually embedded.

The construction for Corollary 1.4 relies on techniques developed by PrzytyckiWise [PW1, PW2, PW3], which can be used to produce closed or bounded virtually essentially embedded subsurfaces of 3-manifolds that intersect JSJ tori in certain controllable pattern. These so-called partial PW subsurfaces have been further investigated in [DLW, Section 4]. Understanding in details how such subsurfaces become virtually embedded leads to constructions crucial to the proof of Theorem 1.1.

Theorem 1.1 is inspired by previous works of various authors. In Przytycki-Wise [PW2], we notice that an essentially immersed subsurface without geometrically infinite JSJ subsurfaces carried by hyperbolic pieces is virtually embedded as long as the graph-manifold part is virtually embedded, and the obstruction to virtual embedding lies only in the horizontal part, known to be Rubinstein-Wang's invariant $s$. In Rubinstein-Wang [RW] and Cooper-Long-Reid [CLR1, CLR2], we notice that horizontally immersed subsurfaces of graph manifold and geometrically infinite subsurfaces in hyperbolic 3-manifolds have certain similar behavior, especially if the 3-manifold is fibered and the subsurface is transverse to a canonical suspension flow. Hinted by the observations, we anticipate a uniform treatment for the almost fiber part, and an extension of Przytycki-Wise's work to handle the general situation. By intuition, it would be very surprising if a closed orientable essentially embedded subsurface failed to be virtually taut, or in other words, virtually Thurston norm minimizing. Having observed that fibers or retracts of JSJ pieces are taut subsurfaces, we are able to strengthen the argument for virtual embedding to show virtual tautness.

In Section 2, we review preliminary materials in 3-manifold topology and introduce some terminology. In Sections 3, 4, we define and study the spirality character of the almost fiber part; in Sections 5, 6, we prove the main result Theorem 1.1. In Sections 7, 8, we introduce pseudo graph manifolds, a generalization of graph manifolds containing atoroidal pieces equipped with pseudo-Anosov suspension flow structures. We derive a practical formula to calculate the spirality character for 
subsurfaces transverse to flow in pseudo graph manifolds, and prove Corollaries 1.3, 1.4. In Section 9, we address the decision problem for surface subgroup separability using the spirality character, and prove Corollary 1.2. In Section 10, we propose a few related questions.

Acknowledgement. The author thanks Yi Ni and Alan Reid for valuable communications. The author would also like to thank the anonymous referee for pointing out some incorrect formulations in Proposition 3.1 and Formula 7.2 in an earlier manuscript.

\section{Preliminaries}

In this section, we review JSJ decomposition of 3-manifolds [JS, Joh, Th1] and the Nielsen-Thurston classification of surface automorphisms [Ni, Th2]. We refer to the survey [AFW1] for topics related to virtual properties of 3-manifolds.

2.1. JSJ decomposition. Let $M$ be a compact orientable irreducible 3-manifold. The Jaco-Shalen-Johanson (JSJ) decomposition asserts that $M$ can be cut along a minimal collection of essential tori, canonical up to isotopy, into Seifert fibered pieces and atoroidal pieces. We call the minimal collection of tori the JSJ tori, and the pieces the JSJ pieces. The JSJ decomposition is a graph-of-spaces decomposition of $M$ whose vertices and edges naturally correspond to the JSJ pieces and the JSJ tori, respectively. It induces a graph-of-group decomposition of $\pi_{1}(M)$ up to natural isomorphism with respect to choices of base points.

2.1.1. Structure of JSJ pieces. We say that a compact orientable irreducible 3manifold is nonelementary if its fundamental group is not virtually solvable. By Hyperbolization, a nonelementary atoroidal JSJ piece is $\mathbf{H}^{3}$-geometric, and the hyperbolic structure is unique up to isometry. A nonelementary Seifert fibered JSJ piece is a circle bundle over a hyperbolic 2-orbifold, and the Seifert fibration structure is canonical up to isotopy. When passing to a finite cover of $M$, every nonelementary JSJ piece elevates to be JSJ pieces of the cover. On the other hand, if $M$ is not itself elementary, any elementary JSJ piece of $M$ is homeomorphic to the orientable thickened Klein bottle, namely, the (characteristic) twisted compact interval bundle over a Klein bottle whose total space is orientable. When passing to a finite cover of $M$, an elementary JSJ piece may elevate to be a regular neighborhood of a JSJ torus. In fact, if $M$ is not elementary, there is always a double cover of $M$ in which there are no elementary JSJ pieces.

2.1.2. Induced decomposition on subsurfaces. Let $M$ be an orientable closed aspherical 3-manifold, and $S$ be a closed essentially immersed subsurface. Up to homotopy, $S$ intersects the JSJ tori of $M$ in a minimal finite (possibly empty) collection of disjoint essential curves of $S$, called the JSJ curves, and the complementary components of the union of the JSJ curves are essential subsurface of $S$, called the JSJ subsurfaces. The decomposition of $S$ above induced from the JSJ decomposition of $M$ is unque up to isotopy, which we will refer to as the induced JSJ decomposition. If $S$ is nonelementary, every JSJ subsurface in an elementary JSJ piece is an essential sub-band of $S$. A JSJ subsurface in a nonelementary atoroidal JSJ piece is either geometrically finite or geometrically infinite with respect to the canonical hyperbolic geometry. A JSJ subsurface in a nonelementary Seifert fibered piece is either vertical or horizontal with respect to the canonical Seifert fibration. It is 
now a known fact that any JSJ subsurface can be lifted to be embedded in some finite cover of the carrying JSJ piece.

A properly embedded subsurface of a compact orientable 3-manifold is said to be an (ordinary or semi) fiber, if the 3-manifold is a surface bundle over a 1-orbifold and the subsurface is isotopic to a fiber. The base 1-orbifold can be a circle or an interval if the subsurface is orientable, or a semi-circle or a semi-interval if the subsurface is nonorientable. A virtual fiber is a properly immersed subsurface which can be lifted into some finite cover the 3-manifold to become a fiber up to homotopy relative to boundary. In particular, we have three types of JSJ subsurfaces which are virtual fibers, namely, JSJ subsurfaces in an elementary piece, or geometrically infinite in a nonelementary atoroidal piece, or horizontal in a nonelementary Seifert fibered piece.

Definition 2.1. In the dual graph $\Lambda$ of the induced JSJ decomposition of $S$, the vertices that correspond to the virtual-fiber JSJ subsurfaces of $S$ span a complete subgraph $\Phi(\Lambda)$ of $\Lambda$. The almost fiber part of $S$ is defined to be the essential subsurface

$$
\Phi(S) \subset S
$$

dual to the complete subgraph $\Phi(\Lambda)$.

2.2. Classification of surface automorphisms. Let $F$ be an oriented closed surface of negative Euler characteristic, and $\theta: F \rightarrow F$ be an automorphism, namely, an orientation-preserving homeomorphism. By the Nielsen-Thurston classification of surface automorphisms, there exists a unique minimal collection of essential curves up to isotopy, called the canonical reduction system, such that $\theta$ can be isotoped to preserve a regular neighborhood of the union of the reduction curves, and the first return map to each complementary components is either periodic or pseudo-Anosov up to isotopy. Hence the compact closures of the complement components form two canonical subunions invariant under $\theta$, namely, the periodic part and the pseudo-Anosov part. We denote by $\theta_{\mathrm{per}}$ and $\theta_{\mathrm{pA}}$ the periodic or pseudo-Anosov automorphisms on these parts respectively, which are unique up to topological conjugacy and freely isotopic to the restrictions of $\theta$.

2.2.1. Suspension flows. Denote by $M_{\theta}$ the mapping torus of $\theta$, namely,

$$
M_{\theta}=\frac{F \times[0,1]}{(x, 0) \sim(\theta(x), 1)} .
$$

The JSJ pieces of $M$ are equipped with a canonical flow suspending $\theta_{\mathrm{per}}$ or $\theta_{\mathrm{pA}}$, which we will call the Nielsen-Thurston suspension flow. On each boundary component of a JSJ piece, there exists a closed leaf and different closed leaves are parallel to each other. We will call any such leaf as a degeneracy slope of the JSJ piece, borrowing a term from [GO]. On a Seifert fibered piece, this flow is exactly the Seifert fiberation, and the degeneracy slope is an ordinary fiber. On an atoroidal piece, the flow is a pseudo-Anosov flow, which preserves two singular codimension-1 foliations transverse along flow lines coming from the suspension of the stable and unstable foliations of $\theta_{\mathrm{pA}}$. A JSJ torus $T$ of $M$ receives two degeneracy slopes from the JSJ pieces on both sides, and they do not match with each other in general. 
2.2.2. Fractional Dehn twist coefficients. We introduce a notion of fractional Dehn twist coefficients which generalizes a definition of Honda-Kazez-Matić [HKM]. Given a surface automorphism $\theta: F \rightarrow F$ as above, a sufficiently high power $\theta^{m}$ preserves each reduction curve $e_{1}, \cdots, e_{r}$, and all the periodic points of the restrictions of $\theta_{\mathrm{per}}^{m}$ and $\theta_{\mathrm{pA}}^{m}$ on $e_{1}, \cdots, e_{r}$ are fixed points. Since the restriction of $\theta_{\mathrm{pA}}^{m}$ on the boundary of the pseudo-Anosov part is isotopic to the identity relative to the fixed points, we may extend by the identity and regard $\theta_{\mathrm{pA}}^{m}$ as an automorphism of $F$ up to isotopy. Denote by $D\left(e_{i}\right)$ the (right-hand) Dehn twist of $F$ along $e_{i}$. There is a factorization of $\theta$ into a commutative product

$$
\theta^{m}=D\left(e_{1}\right)^{k_{1}} \cdots D\left(e_{r}\right)^{k_{r}} \theta_{\mathrm{pA}}^{m},
$$

up to isotopy, where $k_{i}$ are integers uniquely determined by $\theta$ and $m$. We define the fractional Dehn twist coefficient of $\theta$ along $c$ to be

$$
\mathbf{c}\left(\theta, e_{i}\right)=k_{i} / m \text {. }
$$

The definition does not depend on the choice of $m$, and indeed, $\mathbf{c}\left(\theta^{n}, e_{i}\right)$ equals $n \cdot \mathbf{c}\left(\theta, e_{i}\right)$. For a reduction curve $e_{i}$ carried by a JSJ torus $T$ of $M_{\theta}$, the fractional Dehn twist coefficient of $\theta$ vanishes along $e_{i}$ exactly when the degeneracy slopes induced from both sides of $T$ match up with each other. Of course, this vanishing occurs only if the reduction curve is adjacent to the pseudo-Anosov part on at least one side.

\section{Spirality of Loops in the almost fiber Part}

We start our discussion with a descriptive definition of spirality, from a dynamical perspective. Let $S$ be a closed essentially immersed subsurface of an orientable closed aspherical 3-manifold $M$.

For a JSJ curve $c$ contained in the almost fiber part $\Phi(S)$, fix a basepoint $*$ in $c$. Then $M$ has an induced basepoint $x_{0}$, and the subgroup $\pi_{1}(S, *)$ of $\pi_{1}\left(M, x_{0}\right)$ gives rise to a covering space $\tilde{M}_{S}$ of $M$ with a lifted basepoint $\tilde{x}_{0}$. Denote by $A_{c}$ the discrete subset of the JSJ cylinder $\tilde{T}$ that carries the based lift of $c$, such that $A_{c}$ consists of the lifts of $x_{0}$ in $\tilde{T}$. Since $\tilde{T}$ is a finitely generated rank- 1 abelian cover of the JSJ torus $T$ of $M$ carrying $c, A_{c}$ can be identified with the deck transformation group, with $\tilde{x}_{0}$ the trivial element. For any closed path $\alpha:[0,1] \rightarrow \Phi(S)$ based at *, the lifts of $\alpha$ based in $A_{c}$ naturally induce a map

$$
\psi_{\alpha}: A_{c} \rightarrow \tilde{M}_{S}
$$

taking any point to the terminal endpoint of the lift of $\alpha$ based at that point. It turns out that the subset of $A_{c}$ :

$$
D_{\alpha}\left(A_{c}\right)=\psi_{\alpha}^{-1}\left(A_{c}\right) \cap A_{c}
$$

contains a finite index subgroup of $A_{c}$. We will see that the system of restrictions of $\psi_{\alpha}$ to $D_{\alpha}\left(A_{c}\right)$ :

$$
\left(\psi_{\alpha}, D_{\alpha}\left(A_{c}\right)\right)
$$

gives rise to a partial action of $\pi_{1}(\Phi(S), *)$ on $A_{c}$. Moreover, we have the following description of the partial transformations $\psi_{\alpha}$ on $A_{c}$ as partial dilatations. See Subsection 3.1 for definitions and elementary properties.

Proposition 3.1. Based lifting induces a canonical partial action of $\pi_{1}(\Phi(S), *)$ on $A_{c}$ by partial dilatations. 
Definition 3.2. The spirality of a closed path $\alpha:[0,1] \rightarrow \Phi(S)$ based at $*$ is defined to be the dilatation rate of the partial transformation $\psi_{\alpha}$ on $A_{c}$, denoted as

$$
s(\alpha)=\lambda\left(\psi_{\alpha}\right) .
$$

Remark 3.3. We will extend the definition to 1-cycles of $\Phi(S)$ (Definition 4.2).

The partial dilatation description of the partial transformations reveals certain asymptotic nature of the spirality. To visualize, let us say that a subsegment of an elevation of $\alpha$ is spiral if it intersects $A_{c}$ in a geometric sequence of points $a, a r, \cdots, a r^{m}$, where $r$ is the spirality of $\alpha$. Then it can be implied from the definition of partial dilatations (Definition 3.4) that there are no bi-infinitely spiral elevations of $\alpha$ in $\tilde{M}_{S}$, but there are always elevations with arbitrarily long spiral segments. When the spirality $s(\alpha)$ is a positive integer, many elevated lines of $\alpha$ in $\tilde{M}_{S}$ are infinitely spiral in one direction toward an end of $\tilde{T}$.

We prove Proposition 3.1 in the rest of this section.

3.1. Partial dilatations. Let $A$ be a finitely generated abelian group of rank at least 1 .

Definition 3.4. A setwise map $\psi: D(A) \rightarrow A$ is called a partial dilatation of $A$ if there exist a pair of nonzero integers $p, q$ such that $D(A)$ contains $q A$, and

$$
\psi(q v)=p v .
$$

holds for all $v \in A$.

Lemma 3.5. The ratio $p / q$ of a partial dilatation $\psi$ does not depend on the choice of defining integers $p, q$.

Proof. Since $D(A)$ contains a finite index subgroup of $A$, we may choose an element $v \in D(A)$ of infinite order. For any defining pairs of integers $(p, q)$ and $\left(p^{\prime}, q^{\prime}\right)$, we have $\psi\left(q q^{\prime} v\right)$ equals $p q^{\prime} v$ and $p^{\prime} q v$, so $p / q$ equals $p^{\prime} / q^{\prime}$.

Definition 3.6. We define the dilatation rate of a partial dilatation $\psi$ of $A$ to be the nonzero rational value

$$
\lambda(\psi)=p / q .
$$

Recall that a right partial action of group $G$ on set $X$ is a collection of pairs $\left(\psi_{g}, D_{g}\right)$ for all $g \in G$, where $D_{g}$ is a subset of $X$ and $\psi_{g}: D_{g} \rightarrow D_{g^{-1}}$ is a bijection, satisfying that $\left(\psi_{\text {id }}, D_{\text {id }}\right)$ is $\left(\operatorname{id}_{X}, X\right)$ and that $D_{g h}$ contains $D_{g} \cap \psi_{g^{-1}}\left(D_{h}\right)$ and $\psi_{g h}$ is an extension of the composed action $\psi_{g}$ followed by $\psi_{h}$, for all $g, h \in G$.

Suppose that $G$ is a group that partially acts on $A$ by partial dilatations $\left(\psi_{g}, D_{g}\right)$. Then it is obvious that the dilatation rate function

$$
\lambda: G \rightarrow \mathbb{Q}^{\times}
$$

which assigns $\lambda\left(\psi_{g}\right)$ to any element $g \in G$ is a group homomorphism.

3.2. The partial action by partial dilatations. Continue to adopt the notations introduced at the beginning of this section.

Lemma 3.7. The system $\left(\psi_{\alpha}, D_{\alpha}\left(A_{c}\right)\right)$ for all $\alpha \in \pi_{1}(\Phi(S), *)$ defines a right partial action of $\pi_{1}(\Phi(S), *)$ on $A_{c}$. 
Proof. For any closed path $\alpha:[0,1] \rightarrow \Phi(S)$ based at $*$, recall that the domain $D_{\alpha}\left(A_{c}\right)$ consists of all the lifts of $x_{0}$ in $A_{c}$ based at which the lift of $\alpha$ arrives into $A_{c}$. By uniqueness of lifting, we have that $\psi_{\alpha}: D_{\alpha}\left(A_{c}\right) \rightarrow D_{\bar{\alpha}}\left(A_{c}\right)$ is bijective, where $\bar{\alpha}$ is the reversal of $\alpha$. Clearly the trivial loop induces the identical transformation on $A_{c}$. Composition of partial transformations $\psi_{\alpha}$, whenever possible, is induced by lifting of concatenations of loops. This shows that $\pi_{1}(\Phi(S), *)$ partially acts on $A_{c}$ from the right.

In order to see that $\pi_{1}(\Phi(S), *)$ partially acts on $A_{c}$ by partial dilatations, we factorize any partial transformation $\psi_{\alpha}$ into the composition of a sequence of partial maps as follows.

Possibly after a homotopy relative to the base point, we may assume that the lifted closed path $\tilde{\alpha}:[0,1] \rightarrow \tilde{M}_{S}$ of $\alpha$ based at $\tilde{x}_{0}$ intersects the JSJ curves of $\Phi(S)$ consecutively in

$$
y_{0}, \cdots, y_{n}
$$

with $y_{0}$ and $y_{n}$ equal to $\tilde{x}_{0}$. Let

$$
\alpha_{1}, \cdots, \alpha_{n}
$$

be the subpaths $\alpha$ so that the corresponding subpaths $\tilde{\alpha}_{i}$ of $\tilde{\alpha}$ are from $y_{i-1}$ to $y_{i}$, respectively. Note that all the $y_{i}$ lie on the lift of $S$ at $\tilde{x}_{0}$. Since each $y_{i}$ lies on a unique lifted JSJ curve $c_{i}$, we denote by $\tilde{T}_{i}$ the JSJ cylinder of $\tilde{M}_{S}$ corresponding to $c_{i}$, not necessarily mutually distinct. Let $A_{i}$ denote the deck transformation group of $\tilde{T}_{i}$ which covers the underlying JSJ torus of $M$. We may identify each $A_{i}$ as the orbit of $y_{i-1}$, so that $a \in A_{i}$ corresponds to $a . y_{i}$. For each $\alpha_{i}$, we obtain an induced map

$$
\psi_{i}: D_{i}\left(A_{i-1}\right) \rightarrow A_{i} .
$$

The domain $D_{i}\left(A_{i-1}\right)$ is the largest subset of $A_{i-1}$ such that the lift of $\alpha_{i}$ based at any point $y \in D_{i}\left(A_{i-1}\right)$ has its terminal endpoint contained in $A_{i-1}$, and terminal endpoint is defined to be $\psi_{i}(y)$. To facilitate the notation, we regard $\left(\psi_{i}, D_{i}\left(A_{i-1}\right)\right)$ as a partial map of $A_{i-1}$ to $A_{i}$, denoted as

$$
\psi_{i}: A_{i-1} \rightarrow A_{i},
$$

with the domain implicitly assumed. The composition of two partial maps is the partial map defined on the largest domain so that the composition is admissible in the obvious sense. With these notations, the maps $\psi_{i}$ give rise to a restricted factorization of $\psi_{\alpha}$ :

$$
A_{0} \stackrel{\psi_{1}}{\rightarrow} A_{1} \stackrel{\psi_{2}}{\rightarrow} \ldots \stackrel{\psi_{n}}{\rightarrow} A_{n},
$$

where $A_{0}$ and $A_{n}$ are both $A_{c}$, in other words, $\psi_{\alpha}$ is an extension of the composition of $\psi_{i}$ with possibly larger domain.

It would be convenient to extend the definition of partial dilatations to partial maps between different groups. We say that a partial map

$$
\psi: A \rightarrow A^{\prime}
$$

between two finitely generated rank-1 abelian groups is a partial dilatation if there exist infinite-order elements $v \in A$ and $v^{\prime} \in A^{\prime}$, so that $D(A)$ contains $\mathbb{Z} v$, and

$$
\psi(m v)=m v^{\prime} .
$$


holds for all $m \in \mathbb{Z}$. Note that the image of $\psi$ contains $\mathbb{Z} v^{\prime}$, so the composition of two partial dilatations is still a partial dilatation. When $A$ equals $A^{\prime}$, it is easy to check that the new definition agrees with the definition before.

Lemma 3.8. Each partial map $\psi_{i}: A_{i-1} \rightarrow A_{i}$ is a partial dilatation.

Proof. Let $S_{v}$ be the JSJ subsurface of $\Phi(S)$ containing the subpath $\alpha_{i}$.

First assume that $S_{v}$ is orientable. Denote by $\tilde{J}_{v}$ the induced JSJ piece of $\tilde{M}_{S}$ which intersects the based lift of $S$ in a lift of $S_{v}$. As $S_{v}$ is a virtual fiber of the carrier JSJ piece $J_{v}$ of $M, \tilde{J}_{v}$ is a virtually infinite cyclic cover of $J_{v}$. Thus there exists a deck transformation $\tau: \tilde{J}_{v} \rightarrow \tilde{J}_{v}$ of infinite order. Moreover, possibly after replacing $\tau$ with a nontrivial power, we may assume $\tau$ to preserve each boundary component of $\tilde{J}_{v}$. In particular, $\tau$ acts on the JSJ cylinders $\tilde{T}_{i-1}$ and $\tilde{T}_{i}$ by infiniteorder deck transformations, which are affine translations by adding $a_{i-1} \in A_{i-1}$ and $a_{i} \in A_{i}$ respectively. In other words, the domain $D_{i}\left(A_{i-1}\right)$ of $\psi_{i}$ contains $\mathbb{Z} a_{i-1}$, and

$$
\psi_{i}\left(m a_{i-1}\right)=m a_{i}
$$

for all $m \in \mathbb{Z}$. Therefore, $\psi_{i}$ is a partial dilatation.

If $S_{v}$ is not orientable, we may take the characteristic orientable double cover $S_{v}^{*}$, namely, the double cover corresponding to the kernel of the orientation character $\omega: \pi_{1}\left(S_{v}\right) \rightarrow\{ \pm 1\}$. The pullback cover $\tilde{J}_{v}^{*}$ of $\tilde{J}_{v}$ is equipped with a free involution $\nu$. As before, we may find an infinite-order deck transformation $\tau^{*}$ for $\tilde{J}_{v}^{*}$ componentwise preserving the boundary. Observe that each component of $\partial \tilde{J}_{v}$ has two disjoint lifts in $\partial \tilde{J}_{v}^{*}$ that are exchanged under $\nu$, so the action of $\tau^{*}$ commutes with $\nu$ on $\partial \tilde{J}_{v}^{*}$. Thus $\tau^{*}$ descends to become an affine translation $\tau$ acting on $\partial \tilde{J}_{v}$, and in particular, on $\tilde{T}_{i-1}$ and $\tilde{T}_{i}$. We may proceed to complete the proof by the same argument as of the orientable case.

By Lemma 3.8, the composition of the partial dilatations $\psi_{1}, \cdots, \psi_{n}$ is also a partial dilatation. Since $\psi_{\alpha}$ is an extension of the composition, $\psi_{\alpha}$ is a partial dilatation as well. This completes the proof of Proposition 3.1.

\section{THE SPIRALity CHARACTER OF THE ALMOST FIBER PART}

In this section, we take a constructive approach and recapture the spriality of the almost fiber part via a canonically associated $\mathbb{Q}^{\times}$-principal bundle. Here $\mathbb{Q}^{\times}$ denotes the multiplicative group of nonzero rational numbers endowed with the discrete topology. The holonomy of a $\mathbb{Q}^{\times}$-principal bundle induces a so-called spirality character in the first cohomology of the base space in $\mathbb{Q}^{\times}$coefficient. See Subsection 4.1 for details. The construction is based on a flexible auxiliary choice of fibered covers of the JSJ pieces which carry JSJ subsurfaces of the almost fiber part, and the setting will be convenient for calculation.

Let $M$ be an orientable closed 3-manifold, and $S$ be a closed essentially immersed subsurface of $M$.

Proposition 4.1. There exists a principal $\mathbb{Q}^{\times}$-bundle over the almost fiber part:

$$
\mathscr{H} \rightarrow \Phi(S)
$$

naturally induced by the immersion up to isomorphism of principal $\mathbb{Q}^{\times}$-bundles, whose spirality character (Definition 4.3) satisfies

$$
\langle s(\mathscr{H}),[\alpha]\rangle=s(\alpha),
$$


for any closed path $\alpha$ in $\Phi(S)$ with a basepoint on a JSJ curve. Moreover, the sign reduction of the spirality character of $\mathscr{H}$ is the orientation character of $\Phi(S)$.

Here the naturality simply means that for any finite covering map $S^{\prime} \rightarrow S$, the pullback of $\mathscr{H} \rightarrow \Phi(S)$ is isomorphic to $\mathscr{H}^{\prime} \rightarrow \Phi\left(S^{\prime}\right)$.

Definition 4.2. The spirality character of the almost fiber part of $S$ is defined to be

$$
s(\mathscr{H}) \in H^{1}\left(\Phi(S) ; \mathbb{Q}^{\times}\right),
$$

namely, the spirality character of the associated principal $\mathbb{Q}^{\times}$-bundle $\mathscr{H}$. For any integral 1-cycle $\alpha$ of $\Phi(S)$, the spirality of $\alpha$ is defined to be

$$
s(\alpha)=\langle s(\mathscr{H}),[\alpha]\rangle,
$$

where $[\alpha] \in H_{1}(\Phi(S) ; \mathbb{Z})$ is the represented homology class.

We prove Proposition 4.1 in the rest of this section.

\subsection{The spirality character.}

Definition 4.3. For a principal $\mathbb{Q}^{\times}$-bundle $P$ over a CW space $X$, we define the spirality character of $P$, denoted as

$$
s(P) \in H^{1}\left(X ; \mathbb{Q}^{\times}\right),
$$

by the holonomy of $P$.

In other words, for any closed path $\alpha:[0,1] \rightarrow X$, after specifying a lift of the initial point, there is a unique lift $\tilde{\alpha}:[0,1] \rightarrow P$ of $\alpha$. The ratio $\tilde{\alpha}(1) / \tilde{\alpha}(0)$ valued in $\mathbb{Q}^{\times}$depends only on $[\alpha] \in H_{1}(X ; \mathbb{Z})$ and induces a homomorphism of abelian groups

$$
s(P): H_{1}(X ; \mathbb{Z}) \rightarrow \mathbb{Q}^{\times} .
$$

We may alternatively regard $s(P)$ as an element of $H^{1}\left(X ; \mathbb{Q}^{\times}\right)$.

The spirality character is a (multiplicatively valued) characteristic class of principal $\mathbb{Q}^{\times}$-bundles in the sense that it is natural with respect to pullbacks. Under the sign homomorphism sgn: $\mathbb{Q}^{\times} \rightarrow\{ \pm 1\}$, the induced homomorphism

$$
\text { sgn : } H^{1}\left(X ; \mathbb{Q}^{\times}\right) \rightarrow H^{1}(X ;\{ \pm 1\})
$$

reduces the spirality character of $P$ to the orientation character of the associated principal $\{ \pm 1\}$-bundle $\operatorname{sgn}(P)=P \times \operatorname{sgn}\{ \pm 1\}$ :

$$
\operatorname{sgn}(s(P))=\omega(\operatorname{sgn}(P)) .
$$

Here $\omega$ denotes the orientation character, which is just the first Stiefel-Whitney class (of an associated real vector bundle), except with the additive coefficient group $\mathbb{Z} / 2$ treated as the multiplicative group $\{ \pm 1\}$ instead.

Definition 4.4. We say that $P$ is aspiral if $s(P)$ is torsion, or equivalently, only takes values \pm 1 for all $[\alpha] \in H_{1}(X ; \mathbb{Z})$.

A general formula to calculate the spirality character will be given based on some topological setup data that we choose to construct of the associated principal bundle, (Formula 4.5).

\subsection{The associated principal bundle $\mathscr{H}$.}


4.2.1. Setup. As $S$ has induced decomposition by the JSJ curves, denote by $\Lambda$ and $\Phi(\Lambda)$ the dual graphs of $S$ and $\Phi(S)$, respectively. We make a choice of setup data as follows.

For each vertex $v$ of $\Phi(\Lambda)$, choose a triple

$$
\left(S_{v}^{\prime}, \phi_{v}^{\prime},\left\{c_{\delta}^{\prime}\right\}_{\delta \mid v}\right)
$$

Here $S_{v}^{\prime}$ is an orientable finite cover of the JSJ subsurface $S_{v}$ corresponding to $v$, and $\phi_{v}^{\prime}$ is an automorphism of $S_{v}^{\prime}$ fixing $\partial S_{v}^{\prime}$, and for each end-of-edge $\delta$ adjacent to $v, c_{\delta}^{\prime}$ is an elevation of the boundary component $c_{\delta}$ of $S_{v}$ corresponding to $\delta$. Implicitly, we also require that the mapping torus

$$
J_{v}^{\prime}=\frac{S_{v}^{\prime} \times[0,1]}{(x, 0) \sim\left(\phi_{v}^{\prime}(x), 1\right)}
$$

is equipped with a finite covering projection to the JSJ piece $J$ of $M$ containing $S_{v}$, taking $S_{v}^{\prime} \times\{0\}$ to $S_{v}$.

4.2.2. The construction. To construct a principal $\mathbb{Q}^{\times}$-bundle over $\Phi(S)$, we start by taking a trivialized principal $\mathbb{Q}^{\times}$-bundle over $S_{v}$ :

$$
\mathscr{H}_{v}^{+} \cong S_{v} \times \mathbb{Q}^{\times}
$$

for each vertex $v \in \Phi(\Lambda)$. To glue these vertex principal $\mathbb{Q}^{\times}$-bundles $\mathscr{H}_{v}^{+}$along the JSJ curves, identify the fibers over $c_{\delta}$ and $c_{\bar{\delta}}$ via a principal-bundle equivalence for any end-of-edge $\delta$ of $\Phi(\Lambda)$, where $\bar{\delta}$ denotes the opposite end of the same edge. Precisely, the gluing is given by a homeomorphism

$$
c_{\delta} \times \mathbb{Q}^{\times} \rightarrow c_{\bar{\delta}} \times \mathbb{Q}^{\times}
$$

which naturally identifies the first factors and multiplies the second factor by a positive rational ratio $s_{\delta}$, satisfying

$$
s_{\bar{\delta}}=s_{\delta}^{-1} .
$$

The ratios $s_{\delta}$ are determined as follows. For each boundary curve $c_{\delta}$ of each JSJ subsurface $S_{v}$, the chosen elevated curve $c_{\delta}^{\prime}$ lies in a boundary component $T_{\delta}^{\prime}$ of $J_{v}^{\prime}$. Denote by $J_{v}$ and $T_{\delta}$ the JSJ piece of $M$ and the boundary component covered by $J_{v}^{\prime}$ and $T_{\delta}^{\prime}$, respectively. We associate to $c_{\delta}$ a nonzero integer

$$
h_{\delta}=\left[T_{\delta}^{\prime}: T_{\delta}\right] /\left[c_{\delta}^{\prime}: c_{\delta}\right],
$$

where $[-:-]$ denotes the covering degree. The ratio $s_{\delta}$ is then defined to be

$$
s_{\delta}=h_{\bar{\delta}} / h_{\delta} .
$$

The construction above, or essentially the ratios $s_{\delta}$, yields a principal $\mathbb{Q}^{\times}$-bundle over the almost fiber part $\Phi(S)$, which we denote as $\mathscr{H}^{+} \rightarrow \Phi(S)$. To correct the orientation character, we further twist $\mathscr{H}^{+}$by taking the fiber product

$$
\mathscr{H}^{+} \times_{\Phi(S)} \tilde{S},
$$

where $\tilde{S} \rightarrow S$ is the orientable double cover corresponding to the kernel of $\omega(S) \in$ $H^{1}(S ;\{ \pm 1\}) \cong \operatorname{Hom}\left(\pi_{1}(S),\{ \pm 1\}\right)$, regarded as a principal $\{ \pm 1\}$-bundle over $\Phi(S)$. The result is the associated principal $\mathbb{Q}^{\times}$-bundle

$$
\mathscr{H} \rightarrow \Phi(S) .
$$


4.2.3. Verifications. In the above setting, by Definition 4.3 , the spirality character of $\mathscr{H}$ can be calculated using the following formula:

Formula 4.5. For any directed 1 -cycle $\alpha$ dual to a cycle of directed edges $e_{1}, \cdots, e_{n}$,

$$
|\langle s(\mathscr{H}),[\alpha]\rangle|=\prod_{i=1}^{n} s_{\operatorname{ter}\left(e_{i}\right)}=\prod_{i=1}^{n} s_{\operatorname{ini}\left(e_{i}\right)}^{-1}=\prod_{i=1}^{n} \frac{h_{\operatorname{ini}\left(e_{i}\right)}}{h_{\operatorname{ter}\left(e_{i}\right)}},
$$

and the sign of $\langle s(\mathscr{H}),[\alpha]\rangle$ is the value of the orientation character of $\Phi(S)$ on $[\alpha]$.

We must verify that for any closed path $\alpha$ with basepoint in a JSJ curve of $\Phi(S)$, $\langle s(\mathscr{H}),[\alpha]\rangle$ equals the dilatation rate $\lambda\left(\psi_{\alpha}\right)$, (Definition 3.2). Once this is done, we can infer immediately that the isomorphism class of the principal $\mathbb{Q}^{\times}$-bundle $\mathscr{H}$ does not depend on the choice of the setup triple $\left(S_{v}^{\prime}, \phi_{v}^{\prime},\left\{c_{\delta}^{\prime}\right\}_{\delta \mid v}\right)$, and that the dilatation rate $\lambda\left(\psi_{\alpha}\right)$ does not depend on the choice of the basepoint of $\alpha$ either.

Denote by $c$ the JSJ curve of $\Phi(S)$ containing the basepoint of $\alpha$, and by $\alpha_{1}, \cdots, \alpha_{n}$ the subsegments in which $\alpha$ intersects with the JSJ subsurfaces of $\Phi(S)$, in cyclic consecutive order. Denote by $e_{i}$ the edges corresponding to the transition points between the segments $\alpha_{i}$ and $\alpha_{i+1}$. By convention, we understand the index $i$ as a residue class modulo $n$. Adopting the notations in the proof of Proposition 3.1, we have a restricted factorization of $\psi_{\alpha}: A_{c} \rightarrow A_{c}$ into a composition of partial dilatations

$$
\stackrel{\psi_{1}}{\rightarrow} A_{1} \stackrel{\psi_{2}}{\rightarrow} \cdots \stackrel{\psi_{n}}{\rightarrow} A_{n}
$$

where $A_{0}$ and $A_{n}$ are both $A_{c}$.

In general, a dilatation rate can be assigned to a partial dilatation $\psi: A \rightarrow A^{\prime}$ up to a sign, and the ambiguity can be resolved by specifying an orientation of $A$ and $A^{\prime}$. To this end, we say ad hoc that a finitely generated rank- 1 abelian group $A$ is oriented if a generator of the free cyclic quotient $\bar{A}$ is specified. With this generator identified to be 1 , we may identify the rational vector space $A \otimes \mathbb{Q}$ with $\mathbb{Q}$. Suppose that $A$ and $A^{\prime}$ are both oriented. With any defining elements $v \in A$ and $v^{\prime} \in A^{\prime}, \psi$ induces a homomorphism of rational vector spaces

$$
\bar{\psi}: A \otimes \mathbb{Q} \rightarrow A^{\prime} \otimes \mathbb{Q},
$$

defined by $\bar{\psi}(v \otimes 1)=v^{\prime} \otimes 1$. Since we have identified both $A \otimes \mathbb{Q}$ and $A^{\prime} \otimes \mathbb{Q}$ with $\mathbb{Q}$, the homomorphism $\bar{\psi}$ is the multiplication by a nonzero rational scalar, denoted as

$$
\lambda(\psi) \in \mathbb{Q}^{\times},
$$

and we define $\lambda(\psi)$ to be the dilatation rate of $\psi$. It is easy to see that $\lambda(\psi)$ depends only on $\psi$ and the orientation of $A$ and $A^{\prime}$. Moreover, the dilatation rate is multiplicative under composition of partial dilatations.

We fix an auxiliary choice of orientation for each $A_{i}$. Then

$$
\lambda\left(\psi_{\alpha}\right)=\lambda\left(\psi_{1}\right) \times \cdots \times \lambda\left(\psi_{n}\right) .
$$

Lemma 4.6. For any setup triple $\left(S_{v}^{\prime}, \phi_{v}^{\prime},\left\{c_{\delta}^{\prime}\right\}_{\delta \mid v}\right)$,

$$
\left|\lambda\left(\psi_{i}\right)\right|=\frac{h_{\text {ini }\left(e_{i}\right)}}{h_{\operatorname{ter}\left(e_{i-1}\right)}} .
$$

Proof. We first assume that $\Phi(S)$ is orientable. Let $d$ be a positive integer divisible by all the covering degrees $\left[c_{\delta}^{\prime}: c_{\delta}\right]$. For the mapping torus $J_{v}^{\prime}$ of $\phi_{v}^{\prime}$, consider the $d$-cyclic cover $J_{v}^{\prime \prime}$ of $J_{v}^{\prime}$ dual to $S_{v}^{\prime}$. Since $\phi_{v}^{\prime}$ is boundary fixing, a closed leaf $b_{\delta}^{\prime}$ of the 
suspension flow on $T_{\delta}^{\prime}$ intersects $c_{\delta}^{\prime}$ in only one point, and the elevation $b_{\delta}^{\prime \prime} \operatorname{covers} b_{\delta}^{\prime}$ of degree $d$. Identify the infinite cyclic cover of $T_{\delta}$ with $\tilde{T}_{\delta}$, the corresponding boundary component of the induced JSJ piece $\tilde{J}_{v}$ of $\tilde{M}_{S}$, then $b_{\text {ini }\left(e_{i}\right)}^{\prime \prime}$ can be regarded as an infinite-order element of $A_{i}$, namely, $b_{\text {ini }\left(e_{i}\right)}^{\prime \prime} . y_{i}$, where $y_{i}$ is the intersection of $\alpha$ with the JSJ curve $c_{i}$. Similarly, $b_{\operatorname{ter}\left(e_{i-1}\right)}^{\prime \prime}$ can be regarded as an element of $A_{i-1}$. By definition, $\lambda\left(\psi_{i}\right)$ can be computed using

$$
\bar{\psi}_{i}\left(b_{\operatorname{ter}\left(e_{i-1}\right)}^{\prime \prime} \otimes 1\right)=b_{\operatorname{ini}\left(e_{i}\right)}^{\prime \prime} \otimes 1,
$$

where $b_{\operatorname{ter}\left(e_{i-1}\right)}^{\prime \prime} \otimes 1$ and $b_{\text {ini }\left(e_{i}\right)}^{\prime \prime} \otimes 1$ are rational numbers under identification. Observe that the elevation $T_{\delta}^{\prime \prime}$ of $T_{\delta}^{\prime}$ covers $T_{\delta}$, and the covering map factors through a characteristic finite cover of $T_{\delta}$ in which $c_{\delta}^{\prime}$ has a lift, by the divisibility of $d$. This implies

and

$$
\frac{\left[T_{\operatorname{ter}\left(e_{i-1}\right)}^{\prime \prime}: T_{\operatorname{ter}\left(e_{i-1}\right)}\right]}{\left[c_{\operatorname{ter}\left(e_{i-1}\right)}^{\prime \prime}: c_{\operatorname{ter}\left(e_{i-1}\right)}\right]}=\left|b_{\operatorname{ter}\left(e_{i-1}\right)}^{\prime \prime} \otimes 1\right|,
$$

$$
\frac{\left[T_{\operatorname{ter}\left(e_{i-1}\right)}^{\prime \prime}: T_{\operatorname{ter}\left(e_{i-1}\right)}\right]}{\left[c_{\operatorname{ter}\left(e_{i-1}\right)}^{\prime \prime}: c_{\operatorname{ter}\left(e_{i-1}\right)}\right]}=d \cdot \frac{\left[T_{\operatorname{ter}\left(e_{i-1}\right)}^{\prime}: T_{\operatorname{ter}\left(e_{i-1}\right)}\right]}{\left[c_{\operatorname{ter}\left(e_{i-1}\right)}^{\prime}: c_{\operatorname{ter}\left(e_{i-1}\right)}\right]} .
$$

The same computation works for the initial endpoint of $e_{i}$ as well. We obtain

$$
\left|\lambda\left(\psi_{i}\right)\right|=\frac{\left[T_{\operatorname{ini}\left(e_{i}\right)}^{\prime \prime}: T_{\operatorname{ini}\left(e_{i}\right)}\right] /\left[c_{\operatorname{ini}\left(e_{i}\right)}^{\prime \prime}: c_{\operatorname{ini}\left(e_{i}\right)}\right]}{\left[T_{\operatorname{ter}\left(e_{i-1}\right)}^{\prime \prime}: T_{\operatorname{ter}\left(e_{i-1}\right)}\right] /\left[c_{\operatorname{ter}\left(e_{i-1}\right)}^{\prime \prime}: c_{\operatorname{ter}\left(e_{i-1}\right)}\right]}=\frac{h_{\operatorname{ini}\left(e_{i}\right)}}{h_{\operatorname{ter}\left(e_{i-1}\right)}} .
$$

If $\Phi(S)$ is not orientable, in order to compute $\lambda\left(\psi_{i}\right)$, we need to work with the orientable double cover $S_{v}^{*}$ for any non-orientable $S_{v}$, and return to the $S_{v}$ level by only restricting to the JSJ cylinders. However, the idea is similar to the proof of 3.8 and the argument is completely similar to the orientable case, so we omit the details.

By Formula 4.5, Lemma 4.6 and Equation (4.5), it follows that $\langle s(\mathscr{H}),[\alpha]\rangle$ equals $\lambda\left(\psi_{\alpha}\right)$ in the absolute value. To check the sign, observe that there are an odd number of negative values among all $\lambda\left(\psi_{i}\right)$ if and only if the orientation character $\omega(\Phi(S))$ is -1 on $\alpha$. Note that $\tilde{M}_{S}$ is orientable and the JSJ cylinders are all two-sided. Thus the sign matches by the fact that $\operatorname{sgn}(s(\mathscr{H}))$ equals $\omega(\Phi(S))$. In particular, the quantity

$$
\langle s(\mathscr{H}),[\alpha]\rangle=\lambda\left(\psi_{\alpha}\right)
$$

is independent of the choice of the defining triple of $\mathscr{H}$ or the loop representative of $[\alpha]$. Thus $\mathscr{H} \rightarrow \Phi(S)$ is canonically defined up to isotopy of $\Phi(S)$ and bundle equivalence.

To see the naturality of $\mathscr{H} \rightarrow \Phi(S)$, consider a finite covering map $\kappa: \tilde{S} \rightarrow S$. The almost fiber part $\Phi(\tilde{S})$ is the preimage of $\Phi(S)$. Choose a collection of setup data $\left(S_{v}^{\prime}, \phi_{v}^{\prime},\left\{c_{\delta}^{\prime}\right\}_{\delta \mid v}\right)$ for the canonical principal bundle $\mathscr{H}_{S} \rightarrow \Phi(S)$, possibly after modification by passing to multiples of $\phi_{v}^{\prime}$, we may choose a collection of setup data $\left(\tilde{S}_{\tilde{v}}^{\prime}, \tilde{\phi}_{\tilde{v}}^{\prime},\left\{\tilde{c}_{\tilde{\delta}}^{\prime}\right\} \tilde{\tilde{\delta} \mid \tilde{v}}\right)$ commutative with the covering map to construct the canonical pricipal bundle $\mathscr{H}_{\tilde{S}} \rightarrow \Phi(\tilde{S})$. Then Formula 4.5 implies that

$$
s\left(\mathscr{H}_{\tilde{S}}\right)=\kappa^{*} s\left(\mathscr{H}_{S}\right),
$$

so $\mathscr{H}_{\tilde{S}}$ is isomorphic to $\kappa^{*} \mathscr{H}_{S}$. This completes the proof of Proposition 4.1. 


\section{Separability of SURfaCe Subgroups}

In this section, we prove a weak version of the aspirality criterion. Namely, we show that aspirality of the almost fiber part implies a virtually embedded elevation. Invoking Przytycki-Wise [PW3], one will infer $\pi_{1}$-separability and the virtual embedding property from aspirality of the almost fiber part.

Proposition 5.1. Let $M$ be a closed orientable aspherical 3-manifold, and $S$ be a closed essentially immersed subsurface. If the almost fiber part $\Phi(S)$ is aspiral, then $S$ has an embedded elevation in some finite cover of $M$.

The rest of this section is devoted to the proof of Proposition 5.1. As the statement is about elevations of $S$, we may assume without loss of generality that $S$ is orientable.

5.1. Semicovers and merging. Finite covers of an orientable irreducible closed 3-manifold can be obtained by merging finite semicovers. We briefly review some available techniques for our application.

With respect to the JSJ decomposition of $M$, a semicover of $M$ is a 3-manifold $N$ together with a closed immersion $N \rightarrow M$ of which the restriction to any component of $\partial N$ covers a JSJ torus $M$, [PW3, Definition 3.3]. A finite semicover is a semicover where $N$ is compact.

The following lemma allows us to merge atomic finite semicovers into a finite cover of a 3-manifold. More effecient operations such as [PW3, Proposition 3.4] can be derived from this basic case. Given a positive integer $m$, we say that a finite semicover $N \rightarrow M$ is JSJ $m$-characteristic, if every elevation $\tilde{T}$ in $N$ of a JSJ torus $T$ in $M$ is an $m$-characteristic cover of $T$, namely, such that every slope of $\tilde{T}$ covers a slope of $T$ with degree $m$.

Lemma 5.2 (Cf. [DLW, Proposition 4.2]). Let $M$ be an orientable irreducible closed 3-manifold. Suppose $J_{1}^{\prime}, \cdots, J_{s}^{\prime}$ are finite covers of all the JSJ pieces $J_{1}, \cdots, J_{s}$ of $M$, respectively. Then there is a positive integer $m_{0}$, satisfying the following. For any positive integral multiple $m$ of $m_{0}$, there is a regular finite cover $\tilde{M}$ of $M$, which is JSJ $m$-characteristic, such that any elevation $\tilde{J}_{i}$ of a JSJ piece $J_{i}$ is a cover of $J_{i}$ that factors through $J_{i}^{\prime}$.

5.2. Aspiral almost fiber part. The following lemma follows the same idea of Rubinstein and Wang [RW, Lemma 2.4].

Lemma 5.3. As $\Phi(S)$ is orientable and aspiral, there exists a finite semicover

$$
Q(\Phi(S)) \rightarrow M
$$

where $Q(\Phi(S))$ is a bundle over the circle, and the restricted immersion $\Phi(S) \hookrightarrow M$ factors through $Q(\Phi(S))$ as a fiber.

Proof. It suffices to construct a component $Q(F)$ of $Q(\Phi(S))$ for each component of $F$ of $\Phi(S)$. We may assume that $F$ contains a JSJ curve $c$ in the interior, otherwise $F$ is already a virtual fiber of the carrier JSJ piece of $M$.

Choose a basepoint $*$ of $S$ in $c$, which is mapped to a basepoint $x_{0}$ of $M$. Let $\left(\tilde{M}_{S}, \tilde{x}_{0}\right)$ be the pointed covering space of $M$ corresponding to the image of $\pi_{1}(S, *)$ in $\pi_{1}\left(M, x_{0}\right)$. We denote by $F_{0}$ the lift of $F$ at $\tilde{x}_{0}$, which is contained in a unique submanifold $\tilde{M}_{F}$ of $\tilde{M}_{S}$ bounded by the JSJ cylinders corresponding to the JSJ curves $\partial F_{0}$ of $S$. 
We claim that there exists a lift $\tilde{F}_{1}$ of $F$ in $\tilde{M}_{F}$ other than $F_{0}$. To see this, we take an auxiliary cell decomposition of $F$ with a base 0-cell *. As $\Phi(S)$ is aspiral, Proposition 3.1 implies that there exists an elevation $\tilde{x}_{1}$ other than $x_{0}$, such that the 1-skeleton of $F$ lifts to $\tilde{M}_{F}$ based at $\tilde{x}_{1}$. In fact, with the notations of Section 3 , if $\alpha_{1}, \cdots, \alpha_{k}$ is a collection of cellular cycles of $F$ based at $*$ which generate $\pi_{1}(F, *)$, then each $\alpha_{i}$ induces a partial map $\phi_{i}: A_{c} \rightarrow A_{c}$ by a partial dilatation, satisfying $\phi_{i}\left(m v_{i}\right)=m v_{i}$ for some $v_{i} \in A_{c}$ and all $m \in \mathbb{Z}$. Possibly after passing to a common multiple, we may assume that all the $v_{i}$ are the same $v \in A_{c}$, so the point $\tilde{x}_{1}$ can be taken as $v \cdot \tilde{x}_{0}$. There is no further obstruction so the lift of the 1-skeleton of $F$ can be extended to be a lift $F_{1}$ at $\tilde{x}_{1}$.

As $F_{0}$ is embedded, it follows that $F_{1}$ and $F_{0}$ are parallel disjoint embeddings by [FHS]. There is a deck transformation $\tau$ of $\tilde{M}_{F}$ taking $F_{0}$ to $F_{1}$. The quotient of $\tilde{M}_{F}$ by the action of $\tau$ is a finite semicover $Q(F)$ of $M$, equipped with the natural immersion $Q(F) \rightarrow M$ induced from the covering map $\tilde{M}_{S} \rightarrow M$. It is clear that $Q(F)$ is a bundle with a fiber $F_{0}$ lifted from $F$, so $Q(F)$ is the component of $Q(\Phi(S))$ as desired.

5.3. Partial suspensions. For any positive integer $m$, we consider an auxiliary space $Y_{m}(S)$, obtained by suspending the augumented almost fiber part of $S$ of degree $m$. The so-called partial suspension $Y_{m}(S)$ is an immersed subcomplex of $M$, extending the immersion of $S$. We will show that $Y_{m}(S)$ can be elevated to be embedded in some finite cover of $M$, as long as $m$ is an integral multiple of some positive integer $m_{0}$ depending on the immersion of $S$. This will imply that $S$ is virtually embedded.

5.3.1. Partial suspensions over the augumented almost fiber part. Since $\Phi(S)$ is orientable and aspiral, by Lemma 5.3, we may take a semicover $Q_{1}(\Phi(S)) \rightarrow M$ through which $\Phi(S)$ factors as a fiber. Furthermore, for each JSJ curve of $S$ disjoint from $\Phi(S)$, we take a finite cover $T_{1}(c)$ of JSJ torus $T(c)$ in which $c$ lifts to be embedded. The disjoint union of $Q_{1}(\Phi(S))$ and all the $T_{1}(c)$, denoted as $Q_{1}\left(\Phi^{*}(S)\right)$, is a fiber bundle over the circle, and any fiber is homeomorphic to the disjoint union of $\Phi(S)$ and all the $c$, denoted as $\Phi^{*}(S)$. There is an induced immersion

$$
Q_{1}\left(\Phi^{*}(S)\right) \rightarrow M .
$$

We will refer to $\Phi^{*}(S)$ as the augumented almost fiber part of $S$, and $Q_{1}\left(\Phi^{*}(S)\right)$ an augumented suspension over $\Phi^{*}(S)$ of degree 1 .

For any positive integer $m$, denote by $Q_{m}\left(\Phi^{*}(S)\right)$ the $m$-cyclic cover of $Q\left(\Phi^{*}(S)\right)$ dual to the fiber $\Phi^{*}(S)$. Denote by

$$
Y_{m}(S)=S \cup_{\Phi^{*}(S)} Q_{m}\left(\Phi^{*}(S)\right)
$$

the space obtained identifying the almost fiber part $\Phi(S)$ of $S$ with a fiber of $Q_{m}\left(\Phi^{*}(S)\right)$. The space $Y_{m}(S)$ is equipped with a locally embedding map:

$$
Y_{m}(S) \rightarrow M .
$$

We will refer to $Y_{m}(S)$ as a partial suspension of $S$ over $\Phi^{*}(S)$ of degree $m$.

5.3.2. Embedded elevations. By saying that a property about the immersion $j$ : $S \leftrightarrow M$ holds for any sufficiently divisible positive integer $m$, we mean that there exists a positive integer $m_{0}$ depending on the immersion, and that the property holds for any positive integer $m$ divisible by $m_{0}$. 
Lemma 5.4. For any sufficiently divisible positive integer $m$, the partial suspension $Y_{m}(S)$ has an embedded elevation in some finite cover of $M$.

Proof. Observe that the partial suspension $Y_{m}(S)$ has an induced JSJ decomposition

$$
Y_{m}(S)=\bigcup_{\left\{Y_{m}\left(c_{e}\right)\right\}}\left\{Y_{m}\left(S_{v}\right)\right\} .
$$

Precisely, every JSJ curve $c_{e}$ of $S$ corresponds to an induced JSJ torus $Y_{m}\left(c_{e}\right)$, the elevation in $Q_{m}\left(\Phi^{*}(S)\right)$ of the JSJ torus of $M$ containing $c_{e}$. Every JSJ surface $S_{v}$ of $S$ corresponds to an induced JSJ piece $Y_{m}\left(S_{v}\right)$ : if $S_{v}$ is contained in $\Phi(S), Y_{m}\left(S_{v}\right)$ is the corresponding component of $Q_{m}(\Phi(S))$; if $S_{v}$ is not contained in $\Phi(S), Y_{m}\left(S_{v}\right)$ is the union of $S_{v}$ and the JSJ tori $Y_{m}\left(c_{\delta}\right)$ for each boundary component $c_{\delta}$ of $\partial S_{v}$. The point of the induced decomposition is that the JSJ pieces corresponding to a non-virtual-fiber JSJ subsurface should not be the subsurface only, but with the hanging adjacent JSJ tori, because these tori are $\pi_{1}$-visible in the carrier JSJ piece of $M$ as well. If one thickens up the immersed $Y_{m}(S)$ in $M$ to be an immersed compact orientable 3-manifold $\mathcal{Y}_{m}(S)$, the induced JSJ decomposition of $\mathcal{Y}_{m}(S)$ in the usual sense will naturally give rise to the induced JSJ decomposition of $Y_{m}(S)$ as described above.

Step 1. For any JSJ piece $Y_{m}\left(S_{v}\right)$, carried by a JSJ piece $J$ of $M$, we claim that for any sufficiently large positive integer $m$, there exists a regular finite cover $J^{\prime}$ of $J$ in which any elevation of $Y_{m}\left(S_{v}\right)$ is embedded.

If $S_{v}$ is contained in $\Phi(S)$, there is nothing to prove, as $Y_{m}\left(S_{v}\right)$ is already a finite cover of $J$. If $S_{v}$ is not contained in $\Phi(S)$, there are two cases: either $S_{v}$ is a properly immersed vertical annulus in a nonelementary Seifert fibered piece $J$, or $S_{v}$ is a properly immersed geometrically finite subsurface in a hyperbolic piece $J$.

When $S_{v}$ is a properly immersed geometrically finite subsurface of a hyperoblic piece $J$, by [PW3, Theorem 4.1], $Y_{m}\left(S_{v}\right)$ is $\pi_{1}$-injective and relatively quasiconvex if $m$ is sufficiently large. Moreover, in this case, it is a consequence of the relative quasiconvex separability due to Wise [Wi, Theorem 16.23] (cf. [PW3, Corollary $4.2])$ that $\pi_{1}\left(Y_{m}\left(S_{v}\right)\right)$ is indeed separable in $\pi_{1}(J)$. Thus, there is a regular finite cover $J^{\prime}$ of $J$ in which any elevation of $Y_{m}\left(S_{v}\right)$ is embedded.

When $S_{v}$ is a properly immersed vertical annulus in a nonelementary Seifert fibered piece $J$, the base 2-orbifold $\mathcal{O}$ is of the hyperbolic type. The JSJ piece $Y_{m}\left(S_{v}\right)$ is a circle bundle over a graph $Z_{m}\left(a_{v}\right)$, where $a_{v}$ is a properly immersed arc in $\mathcal{O}$, and at each endpoint of $a_{v}$ there is a wedged circle covering the corresponding boundary component of $\mathcal{O}$ of some degree divisible by $m$. A direct argument in hyperbolic geometry, or a 2-dimensional version of the argument in the previous case, implies that $\mathcal{O}$ has a regular finite cover $\mathcal{O}^{\prime}$ in which any elevation of $Z_{m}\left(a_{v}\right)$ is embedded. The base cover $\mathcal{O}^{\prime}$ of $\mathcal{O}$ induces a regular finite cover $J^{\prime}$ of $J$, in which any elevation of $Y_{m}\left(S_{v}\right)$ is embedded.

This proves the claim of Step 1.

Step 2. We claim that for any sufficiently divisible integer $m$, there exists a regular finite cover $M^{\prime}$ of $M$ in which all the elevations of the JSJ pieces of $Y_{m}(S)$ are embedded.

We apply the merge trick. We may assume that $m$ is sufficiently large so that the claim of Step 1 holds for all the JSJ pieces $Y_{m}\left(S_{v}\right)$, giving rise to regular finite covers $J_{v}^{\prime}$ for each carrier JSJ piece of $M$. If some of these $J_{v}^{\prime}$ cover the same JSJ 
piece $J$ of $M$, we pass to a common regular finite cover $J^{\prime}$ of these $J_{v}^{\prime}$; if some JSJ piece $J$ of $M$ is not covered by any of these $J_{v}^{\prime}$, we choose $J^{\prime}$ to be $J$ itself. By Lemma 5.2, there exists a regular finite cover $M^{\prime}$ of $M$ merging all these covers $J^{\prime}$ of $J$. Note the conclusion in the claim of Step 1 is not affected passing to further regular finite cover $J^{\prime \prime}$ of $J$ that factors through $J^{\prime}$. Thus, in the regular cover $M^{\prime}$ of $M$, all the elevations of the JSJ pieces of $Y_{m}(S)$ are embedded, as claimed.

Step 3. We finish the proof by showing that the regular finite cover $M^{\prime}$ of $M$ given by Step 2 has a further finite cover $\tilde{M}$ in which $Y_{m}(S)$ has an embedded elevation. Indeed, $\tilde{M}$ will be induced from a finite cover of the dual graph of $M^{\prime}$.

In fact, if $M^{\prime}$ is a regular finite cover of $M$ given by Step 2, any elevation

$$
Y_{m}^{\prime}(S) \rightarrow M^{\prime}
$$

induces a map between the graphs dual to the decomposition

$$
\Lambda\left(Y_{m}^{\prime}(S)\right) \rightarrow \Lambda\left(M^{\prime}\right)
$$

Because the edge spaces of $Y_{m}^{\prime}(S)$ are all finite covers of JSJ tori of $M^{\prime}$, and because each vertex space of $Y_{m}^{\prime}(S)$ is an elevated JSJ piece embedded in a JSJ piece of $M^{\prime}$, the induced map between the dual graphs is a combinatorial local isometry, which is, in particular, $\pi_{1}$-injective. Because $\pi_{1}\left(\Lambda\left(M^{\prime}\right)\right)$ is a free group, and hence is LERF by Scott [Sc], $\Lambda\left(M^{\prime}\right)$ has a regular finite cover in which any elevation $\Lambda\left(Y_{m}^{\prime}(S)\right)$ is an embedded subgraph. Therefore, in the induced regular finite cover $\tilde{M}$ of $M$, any elevation of $Y_{m}(S)$ is embedded.

This completes the proof.

Because the augumented partial suspension $Y_{m}(S)$ contains a $\pi_{1}$-injective embedded copy of $S$, Lemma 5.4 implies that $S$ has an embedded elevation in a finite cover of $M$. This completes the proof of Proposition 5.1.

\section{Virtual tautness}

In this section, we prove virtual tautness of essentially embedded orientable subsurfaces. Then we summarize the proof of Theorem 1.1 in the end of this section.

Recall that the complexity $\chi_{-}(S)$ of a (possibly disconnected) compact orientable surface $S$ is defined to be the opposite of the sum of the negative Euler characteristics of its components. In [Th2], Thurston introduces a semi-norm on $H_{2}(M, \partial M ; \mathbb{Q})$ for any compact 3-manifold $M$, known as the Thurston norm. The Thurston norm $x(\xi)$ of an integral relative second homology class $\xi$ is defined to be the minimum of $\chi_{-}(S)$ among all compact oriented properly embedded subsurfaces $S$ of $M$ representing $\xi$. We say that an oriented (possibly disconnected) compact properly embedded subsurface of a compact 3-manifold is taut if it realizes the Thurston norm of its homology class.

Proposition 6.1. Let $M$ be an orientable closed aspherical 3-manifold, and $S$ be an oriented closed essentially embedded subsurface of $M$. Then $S$ is virtually taut.

The proof of Proposition 6.1 follows the idea in the proof of Proposition 5.1, with strengthened arguments at a number of places. 
6.1. Completing a semicover. We first need a stronger version of PrzytyckiWise [PW3, Proposition 3.4], so as to complete a connected semicover without passing to a further finite cover.

Lemma 6.2. If $N$ is a connected finite semicover of an orientable closed aspherical 3-manifold $M$, then $N$ has an embedded lift in a finite cover of $M$. In fact, the semi-covering $N \rightarrow M$ is $\pi_{1}$-injective and $\pi_{1}$-separable.

Proof. After choosing auxiliary basepoints, we prove that $\pi_{1}(N)$ injects to be a separable subgroup of $\pi_{1}(M)$. Then $N$ is virtually embedded by Scott [Sc, Lemma 1.4]. Because $N$ has an elevation embedded in a finite cover of $M$, [PW3, Proposition 3.4], and separability is a virtual property, we may passing the pair and assume $N$ is embedded in $M$. Choose a basepoint $*$ of $M$ contained in $N$. Below we abuse the notation of pointed loops and elements in fundamental groups.

For any element $\alpha \in \pi_{1}(M)$ not contained in $\pi_{1}(N), \alpha$ is pointed homotopic to a concatenation $\tau \gamma \bar{\tau}$, where $\gamma$ is a loop freely homotopic to $\alpha$, and $\tau$ is a path from * to a point on $\gamma$. Moreover, we require the 1-complex $\gamma \cup \tau$ to be transverse to the JSJ tori, minimizing the number of intersections subject to the conditions above. Because each JSJ torus is $\pi_{1}$-separable in an adjacent JSJ piece [LoN, Theorem 1], for every embedded subpath $\gamma \cup \tau$ which is properly embedded in a JSJ piece of $M$, there is a regular finite cover of the JSJ piece so that any elevation of the path connects two distinct boundary components. Applying merging (Lemma 5.2), we obtain a regular finite pointed cover $M^{\prime}$ of $M$ with a pointed elevation $N^{\prime}$ of $N$. Note that $\pi_{1}\left(N^{\prime}\right)$ equals $\pi_{1}(N) \cap \pi_{1}\left(M^{\prime}\right)$. For any power $\alpha^{\prime}$ of $\alpha$ contained in $\pi_{1}\left(M^{\prime}\right), \alpha^{\prime}$ takes the form $\tau^{\prime} \gamma^{\prime} \bar{\tau}^{\prime}$ up to pointed homotopy, where $\gamma^{\prime}$ is a finite cyclic cover of $\gamma$, and $\tau^{\prime}$ is the pointed lift of $\tau$. The construction of $M^{\prime}$ implies that the projection of the pointed 1-complex $\gamma^{\prime} \cup \tau^{\prime}$ to pointed JSJ dual graph $\Lambda_{M^{\prime}}$ of $M^{\prime}$ is a local embedding. Moreover, since $\alpha \notin \pi_{1}(N)$ and hence $\alpha^{\prime} \notin \pi_{1}\left(N^{\prime}\right)$, the projection of $\gamma^{\prime} \cup \tau^{\prime}$ cannot be contained in the subgraph $\Lambda_{N^{\prime}}$ dual to $N^{\prime}$. Using

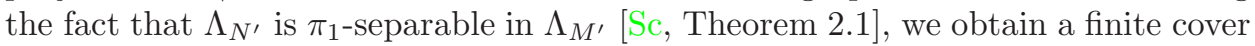
$M^{\prime \prime}$ of $M^{\prime}$ induced by a cover of $\Lambda_{M^{\prime}}$, such that $\pi_{1}\left(N^{\prime}\right)$ is contained in $\pi_{1}\left(M^{\prime \prime}\right)$ but $\alpha^{\prime} \notin \pi_{1}\left(M^{\prime \prime}\right)$, or in other words, $\alpha^{\prime}$ is separable from $\pi_{1}\left(N^{\prime}\right)$. We conclude that $\alpha$ is separable from $\pi_{1}(N)$ in $\pi_{1}(M)$.

6.2. Criteria for tautness. Next, we need to introduce some controllable conditions to guarantee tautness of $S$ in terms of its induced JSJ decomposition.

Lemma 6.3. If each JSJ subsurface of $S$ is taut and each JSJ piece of $M$ contains at most one JSJ subsurface of $S$, then $S$ is taut.

Proof. Let $S^{\prime}$ be a taut subsurface representing $[S] \in H_{2}(J ; \mathbb{Q})$, then $S^{\prime}$ can be isotoped to intersect the JSJ tori of $M$ in directly parallel essential curves. Note that for each JSJ piece $J,[S \cap J]$ and $\left[S^{\prime} \cap J\right]$ represents the same homology class in $H_{2}(J, \partial J)$ by excision. Since the JSJ subsurfaces $S_{v}$ of $S$ are taut and contained by distinct JSJ pieces of $M$,

$$
x([S])=\chi_{-}\left(S^{\prime}\right)=\sum_{J} \chi_{-}\left(S^{\prime} \cap J\right) \geq \sum_{v} \chi_{-}\left(S_{v}\right)=\chi_{-}(S) \geq x([S]) .
$$

Hence $x([S])$ equals $\chi_{-}(S)$, which means $S$ is taut.

Lemma 6.4. If a JSJ subsurface $S_{v}$ contained in a JSJ piece $J$ is either a fiber or a retract of $J$, then $S_{v}$ is taut in $J$. 
Proof. If $S_{v}$ is a fiber of $J$, it is taut because it is a compact leaf of the taut codimension-1 foliation of $J$ given by the bundle structure.

If $S_{v}$ is a retract of $J$, consider any other taut subsurface $E$ representing $\left[S_{v}\right]$ in $H_{2}(J, \partial J ; \mathbb{Q})$. Possibly after gluing indirectly parallel pairs of boundary components, which does not affect the complexity, we may assume for each boundary component $T$ of $J$ that $S_{v} \cap T$ and $E \cap T$ are directly parallel curves of the same multiplicity, possibly zero. The existence of the retraction $J \rightarrow S_{v}$ implies that $S_{v} \cap T$ must either be connected or empty. Moreover, the retraction induces a proper degree one map $E \rightarrow S_{v}$, so

$$
x\left(\left[S_{v}\right]\right)=\chi_{-}(E) \geq \chi_{-}\left(S_{v}\right) \geq x\left(\left[S_{v}\right]\right) .
$$

Hence $S_{v}$ is taut in $J$.

6.3. Virtual embedding of partial suspensions. We modify some argument in the construction of partial suspensions in Subsection 5.3 to fit the assumptions of Lemmas 6.3, 6.4. Adopt the notations there.

Lemma 6.5. Let $S_{v}$ be a JSJ subsurface of $S$ carried by a JSJ piece of $M$. For any sufficiently divisible positive integer $m$, the following conditions are satisfied.

(1) If $S_{v}$ is contained in $\Phi(S)$, then $Y_{m}\left(S_{v}\right)$ is a finite cover $\tilde{J}$ of $J$, and $S_{v}$ intersects each boundary component of $Y_{m}\left(S_{v}\right)$ in a curve;

(2) If $S_{v}$ is not contained in $\Phi(S)$, then some finite cover $\tilde{J}$ of $J$ contains an embedded lift of $Y_{m}\left(S_{v}\right)$, and hence $S_{v}$, as a retract.

Proof. If $S_{v}$ is contained in $\Phi(S), Y_{1}\left(S_{v}\right)$, which is $Q_{1}\left(S_{v}\right)$, can be taken to be $J$. Since $S_{v}$ is oriented and embedded, $Y_{m}\left(S_{v}\right)$ is the $m$-cyclic cover dual to $S_{v}$ of $J$, so for any sufficiently divisible positive integer, $S_{v}$ intersects each boundary of $Y$ in a curve.

If $S_{v}$ is not contained in $\Phi(S), S_{v}$ is either geometrically finite or vertical. In the former case, we strengthen the argument before by invoking Wise [Wi, Theorem 16.23], and conclude that $\pi_{1}\left(Y_{m}\left(S_{v}\right)\right)$ is a virtual retract of $\pi_{1}(J)$. This implies a finite cover $\tilde{J}$ in which $Y_{m}\left(S_{v}\right)$ lifts, and the $\pi_{1}$-retraction map $\tilde{J} \rightarrow Y_{m}\left(S_{v}\right)$ can be constructed by extending the identity map restricted to the lift of $Y_{m}\left(S_{v}\right)$. In the latter case, we may similarly strengthen the argument before to conclude that $Y_{m}\left(S_{v}\right)$ is a virtual retract of $J$, so we omit the details here. Note that $S_{v}$ is also a virtual retract of $J$ as it is a retract of $Y_{m}\left(S_{v}\right)$.

To prove Proposition 6.1, we take a finite cover $\tilde{J}_{v}$ of the JSJ piece of $M$ carrying $S_{v}$, for some sufficiently divisible positive integer $m$ satisfying the conclusion of Lemma 6.5 , The finite covers $\tilde{J}_{v}$ contain embedded lifts of $Y_{m}\left(S_{v}\right)$, so we glue these $\tilde{J}_{v}$ up along some boundary components in the same way we assemble the augumented partial suspension $Y_{m}(S)$ from $Y_{m}\left(S_{v}\right)$. The result is a connected finite semicover $N$ of $M$ in which $Y_{m}(S)$ lifts. By Lemma 6.2, we complete $N$ as a finite cover $\tilde{M}$ of $M$. The finite cover contains a lift of $Y_{m}(S)$, and hence a lift of $S$ satisfying the conditions of the tautness criteria Lemmas 6.3, 6.4. It follows that $S$ lifts to be a taut subsurface of $\tilde{M}$.

This completes the proof of Proposition 6.1.

6.4. The proof of Theorem 1.1. Let $S$ be a closed essentially immersed subsurface of a closed orientable aspherical 3-manifold $M$. 
$(1) \Rightarrow(2)$ : If the almost fiber part $\Phi(S)$ of $S$ is aspiral, by Proposition 5.1, $S$ has a virtually embedded elevation. The latter implies that $S$ is $\pi_{1}$-separable by Przytycki-Wise [PW3], or that $S$ is virtually embedded [Sc, Lemma 1.4].

$(2) \Rightarrow(3)$ : If $S$ is virtually embedded, we may pass to a finite cover and assume that $S$ is already embedded. First suppose that $S$ is orientable. By Proposition 6.1, $S$ is taut in some finite cover $\tilde{M}$ of $M$. In this case, Gabai [Ga] constructs a taut foliation $\mathscr{F}$ of $\tilde{M}$ that contains $S$ as a leaf. In fact, $\mathscr{F}$ can be either finite depth or smooth.

When $S$ is not orientable, we derive a virtual taut foliation from the orientable case as follows. The boundary of a compact regular neighborhood $\mathcal{N}$ of $S$ in $M$ is a twisted interval bundle over $S$ whose boundary is isomorphic to the characteristic orientable double cover $S^{*}$ of $S$, equipped with an orientation-reversing free involution $\nu: S^{*} \rightarrow S^{*}$ which is the deck transformation. As $S^{*}$ is essentially embedded and orientable, the previous case yields a finite cover $M^{\prime}$ in which $S^{*}$ is a leaf of a taut foliation $\mathscr{F}^{*}$. Let $M^{\prime}(S)$ be the compact 3-manifold obtained from $M^{\prime}$ by removing a regular neighborhood of $S^{*}$, so $\partial M^{\prime}(S)$ is homeomorphic to $\left(+S^{*}\right) \sqcup\left(-S^{*}\right)$, two oppositely oriented copies of $S^{*}$. Glue $+S^{*}$ to itself by $\nu$ and similarly for $-S^{*}$. The result is a 3-manifold $\tilde{M}$ which finitely covers $M$ in an obvious way. Moreover, the quotient of $\pm S^{*}$ are two copies of $S$ in $\tilde{M}$, and the complement can be foliated by the leaves of $\mathscr{F}^{*}$. The result is clearly a taut foliation $\mathscr{F}$ of $\tilde{M}$, which contains a lift of $S$ as a leaf, so we have proved the non-orientable case.

$(3) \Rightarrow(1)$ : If $S$ is a compact leaf of a taut foliation of $M$. Possibly after passing to a finite cover of $M$ and an elevation of $S$, we may assume without loss of generality that $S$ is orientable, by the naturality of spirality (Proposition 4.1). Since $[S] \in$ $H_{2}(M ; \mathbb{Z})$ is nontrivial, possibly after passing to a sufficiently divisible finite cyclic cover of $M$ dual to $S$, we may assume that each JSJ curve of $S$ lies on a distinct JSJ torus of $M$. Then Formula 4.5 immediately implies that the spirality of $\Phi(S)$ is trivial. Therefore, $S$ is aspiral in the almost fiber part.

This completes the proof of Theorem 1.1.

\section{IMMERSED SUBSURFACES TRANSVERSE TO SUSPENSION FLOW}

In this section, we derive a formula to calculate the spirality for immersed subsurfaces transverse to suspension flows, which generalizes the formula of RubinsteinWang [RW] in the graph manifold case. We apply the formula to prove Corollary 1.3 in the end of this section.

Definition 7.1. An orientable closed aspherical 3-manifold is said to be a pseudo graph manifold, if every JSJ piece is enriched with a Nielsen-Thurston supsension flow. In other words, each JSJ piece is homeomorphic to the mapping torus of an automorphism of a compact orientable surface which is either periodic or pseudoAnosov, and the flow structure of the JSJ piece is the pullback of the suspension flow. The degeneracy slope of a JSJ piece of $M$ is a closed leaf on a boundary component, which is unique up to isotopy.

Let $c$ be a transversely sided immersed loop carried by a JSJ torus $T$ of $M$, namely, $c$ is immersed in $T$ and assigned with a side of $T$. Denote by $l^{ \pm}$the 
degeneracy slopes of $T$ induced from the \pm side of $c$. We define

$$
\sigma_{M}(c)=\frac{\mathbf{i}\left(c, l^{-}\right)}{\mathbf{i}\left(c, l^{+}\right)}
$$

where $\mathbf{i}\left(c, l^{ \pm}\right)$denotes the (minimal) geometric intersection number between $c$ and $l^{ \pm}$.

On the other hand, let $\gamma$ be a directed path in a JSJ piece $J$ of $M$ joining two JSJ torus $T_{\text {ini }(\gamma)}$ and $T_{\operatorname{ter}(\gamma)}$ on the boundary. Denote by $l_{\text {ini }(\gamma)}$ and $l_{\operatorname{ter}(\gamma)}$ the degeneracy slopes respectively. We define

$$
\rho_{M}(\gamma)=\frac{\ell\left(l_{\operatorname{ini}(\gamma)}\right)}{\ell\left(l_{\operatorname{ter}(\gamma)}\right)}
$$

where $\ell$ denotes the length measured in the flowing time unit. Note that $\rho_{M}(\gamma)$ does not change under rescale of flow.

Formula 7.2. Let $M$ be an orientable closed aspherical pseudo graph manifold, and $S$ be an orientable closed essentially immersed subsurface. Suppose that the almost fiber part $\Phi(S)$ is transverse to the JSJ tori and the Nielsen-Thurston flow on the JSJ pieces of $M$. For any immersed directed loop $\alpha$ in $\Phi(S)$ transversely intersecting the (possibly repeating) JSJ curves $c_{1}, \cdots, c_{n}$, denote by $\alpha_{i}$ the subpath joining $c_{i-1}$ and $c_{i}$ with the index understood modulo $n$, and consider $c_{i}$ to be transversely sided in the direction of $\alpha$. The spirality of $\alpha$ is a product:

$$
s(\alpha)=\sigma_{M}(\alpha) \rho_{M}(\alpha)
$$

where

$$
\sigma_{M}(\alpha)=\sigma_{M}\left(c_{1}\right) \times \cdots \times \sigma_{M}\left(c_{n}\right)
$$

and

$$
\rho_{M}(\alpha)=\rho_{M}\left(\alpha_{1}\right) \times \cdots \times \rho_{M}\left(\alpha_{n}\right) .
$$

For example, if $M$ is a graph manifold and $S$ is horizontally immersed, we recover the original formula of Rubinstein-Wang [RW]. Perhaps the most similar situation for a general $M$ is when $S$ is an almost fiber homologically lying in a fibered cone, and is transverse to a canonically induced suspension flow.

Lemma 7.3. If every hyperbolic JSJ piece of $M$ is the mapping torus of a pseudoAnosov automorphism of a compact orientable surface which fixes all the periodic points on the boundary, then:

$$
\rho_{M}(\alpha)=1
$$

Proof. In this case, the length of degeneracy slopes on the boundary of a hyperbolic JSJ piece depends only on the piece. On the other hand, the degeneracy slopes on the boundary of a Seifert fibered piece is necessarily an ordinary fiber, so their length depends only on the piece as well. It follows that each $\rho_{M}\left(\alpha_{i}\right)$ equals 1 so $\rho_{M}(\alpha)$ equals 1 .

Remark 7.4. In general, the correction factor $\rho_{M}$ can be nontrivial since orbit length of periodic points on the boundary for pseudo-Anosov maps may differ as the component varies. For explicit examples, see the pseudo-Anosov braids $\beta_{m, n}$ in $[\mathrm{HK}]$. 
In order to derive the Formula 7.2 from Formula 4.5, we take a setup triple $\left(S_{v}^{\prime}, \phi_{v}^{\prime},\left\{c_{\delta}^{\prime}\right\}_{\delta \mid v}\right)$ as follows. For each JSJ subsurface $S_{v}$ of $\Phi(S)$, take $S_{v}^{\prime}$ to be $S_{v}$, and hence $c_{\delta}^{\prime}$ to be $c_{\delta}$. Denote by $J_{v}$ the JSJ piece of $M$ carrying $S_{v}$, and take $J_{v}^{\prime}$ to be a finite cover of $J_{v}$ with a fiber $S_{v}^{\prime}$ and a boundary-fixing monodromy $\phi_{v}^{\prime}$. Note that $\phi_{v}^{\prime}$ can be freely isotopic to the Nielsen-Thurston normal form which is either periodic or pseudo-Anosov, unique up to conjugacy. Possibly after passing to a power of $\phi_{v}^{\prime}$, we require all the periodic points of the Nielsen-Thurston normal form of $\phi_{v}^{\prime}$ on $\partial S_{v}^{\prime}$ to be fixed by $\phi_{v}^{\prime}$.

The assumption that $\Phi(S)$ is flow-transversely immersed guarantees that the suspension flow of the Nielsen-Thurston normal form of $\phi_{v}^{\prime}$ on $J_{v}^{\prime}$ is conjugate to the pullback of the flow of $J_{v}$. In fact, if $S_{v}$ is carried by a Seifert-fibered piece, $S_{v}$ is horizontally immersed, and the flows are both conjugate to the Seifert fibration of $J_{v}^{\prime}$. If $J_{v}$ is atoroidal, the result is due to Fried [Fr, Theorem 7], cf. McMullen [mM, Theorem 3.1]. In particular, any degeneracy slope $l_{\delta}^{\prime}$ of $J_{v}^{\prime}$ covers the degeneracy slope $l_{\delta}$ of $J_{v}$ up to homotopy. Therefore, the covering degrees between degeneracy slopes can be computed by:

$$
\left[l_{\delta}^{\prime}: l_{\delta}\right]=\ell\left(l_{\delta}^{\prime}\right) / \ell\left(l_{\delta}\right)
$$

For any boundary component $c_{\delta}$ of $S_{v}$ carried by a boundary component $T_{\delta}$ of $J_{v}, c_{\delta}^{\prime}$ is a lift. We have

$$
\left[c_{\delta}^{\prime}: c_{\delta}\right]=1
$$

and

$$
\left[T_{\delta}^{\prime}: T_{\delta}\right]=\left[l_{\delta}^{\prime}: l_{\delta}\right] \cdot\left[c_{\delta}^{\prime}: c_{\delta}\right] \cdot \frac{\mathbf{i}\left(c_{\delta}, l_{\delta}\right)}{\mathbf{i}\left(c_{\delta}^{\prime}, l_{\delta}^{\prime}\right)}=\frac{\mathbf{i}\left(c_{\delta}, l_{\delta}\right) / \ell\left(l_{\delta}\right)}{\mathbf{i}\left(c_{\delta}^{\prime}, l_{\delta}^{\prime}\right) / \ell\left(l_{\delta}^{\prime}\right)} .
$$

Observe that $\mathbf{i}\left(c_{\delta}^{\prime}, l_{\delta}^{\prime}\right)$ equals 1 on $T_{\delta}^{\prime}$ and $\ell\left(l_{\delta}^{\prime}\right)$ depends only on the carrying vertex $v$, by our assumption that the Nielsen-Thurston normal form of $\phi_{v}^{\prime}$ fixes all periodic points on the boundary. Thus the factors on the denominator are cyclically cancelled when plugged into Formula 4.5. Now Formula 7.2 can be deduced Formula 4.5 by simple substitution.

Proof of Corollary 1.3. Let $\theta: F \rightarrow F$ be an orientation-preserving homeomorphism of an oriented closed surface $F$ of negative Euler characteristic. Let $S$ be an oriented closed essentially immersed subsurface of the mapping torus $M_{\theta}$ transverse to the JSJ tori and to the Nielsen-Thurston suspension flow supported in the JSJ pieces.

Suppose that the monodromy $\theta$ has no nontrivial fractional Dehn twist coefficient. It suffices to show that some elevation of $S$ in a finite cover of $M_{\theta}$ is aspiral in the almost fiber part, by Theorem 1.1. By passing to a power of $\theta$, we may assume that $\theta$ fixes all the periodic points on the boundary of the pseudo-Anosov part. For any loop $\alpha$ in the almost fiber part of $S$, we have $\rho_{M_{\theta}}(\alpha)$ equals 1 by Lemma 7.3. Because $\theta$ has no nontrivial fractional Dehn twist coefficient, for all JSJ tori of $M_{\theta}$, the degeneracy slopes induced from both sides coincide with each other. Hence $\sigma_{M_{\theta}}(\alpha)$ equals 1. By Formula 7.2 we conclude that $S$ is aspiral in the almost fiber part.

\section{Application}

In this section, we exhibit new examples of subsurfaces essentially immersed but not virtually embedded, as asserted by Corollary 1.4. We construct the subsurface 
by applying techniques developed in [PW1, PW2, DLW], and detect a loop with nontrivial spirality using Formula 7.2.

Example 8.1. Let $M_{\theta}$ be a closed orientable surface bundle fibering over the circle with a fiber $F$ and a monodromy $\theta: F \rightarrow F$. Suppose that $\theta$ has a nontrivial fractional Dehn twist coefficient along a reduction curve $e$. We construct a closed essentially immersed subsurface $S$ of $M_{\theta}$ which is not virtually embedded.

The idea is first to construct locally an essentially immersed almost fiber subsurface $E$, within the JSJ pieces adjacent to the carrier JSJ torus $e$. We carefully make sure that $E$ has nontrivial spirality using the fact that the fractional Dehn twist coefficient of $\theta$ along $e$ is nontrivial. Take two oppositely oriented copies $E$ and $\bar{E}$. Then, we can merge them along boundary with other virtually embedded subsurfaces to obtain a closed subsurface of the 3-manifold. Below we give an outline of a construction.

Construction. Rewrite $M_{\theta}$ as $M$ for simplicity, and denote by $T$ the JSJ torus carrying $e$. Possibly after passing to a finite cover of $M$ and an elevation of $F$, we may assume that the $\theta$ preserves all the reduction curves componentwise and the periodic or pseudo-Anosov normal forms in the rest regions fix all periodic points on the boundary. We may also assume that the JSJ torus $T$ is adjacent two distinct JSJ pieces $J^{ \pm}$. Denote by $l^{ \pm}$the degeneracy slopes on $T$ induced from $J^{ \pm}$, directed in the flow direction, and by $F^{ \pm}$the fiber $F \cap J^{ \pm}$. We have

$$
\mathbf{i}\left(e, l^{ \pm}\right)=1,
$$

and in $H_{1}(T ; \mathbb{Z})$, choosing a suitable direction of $e$,

$$
\left[l^{+}\right]-\left[l^{-}\right]=k \cdot[e],
$$

where $k$ is the fractional Dehn twist coefficient of $\theta$, a nonzero integer under our assumption of simplification.

Take a regular JSJ characteristic finite cover of $J^{ \pm}$, denoted by $\tilde{J}^{ \pm}$, such that $\tilde{J}^{ \pm}$ has at least three boundary components covering T, (cf. [CLR3] and Subsection 5.1). Then there exists two boundary components $\tilde{T}_{0}^{ \pm}$and $\tilde{T}_{1}^{ \pm}$of $\tilde{J}^{ \pm}$covering $T$ such that $H_{1}\left(T_{0}^{ \pm} ; \mathbb{Q}\right) \oplus H_{1}\left(T_{1}^{ \pm} ; \mathbb{Q}\right)$ has at least 3 dimensions surviving in $H_{1}\left(\tilde{J}^{ \pm} ; \mathbb{Q}\right)$. Without loss of generality, we may assume $H_{1}\left(T_{1}^{ \pm} ; \mathbb{Q}\right)$ to be embedded in $H_{1}\left(\tilde{J}^{ \pm} ; \mathbb{Q}\right)$. For $i$ equal to 0 or 1 , denote by

$$
\partial_{i}: H_{2}\left(\tilde{J}^{ \pm}, \partial \tilde{J}^{ \pm} ; \mathbb{Z}\right) \rightarrow H_{1}\left(\tilde{T}_{i}^{ \pm} ; \mathbb{Z}\right)
$$

the boundary homomorphism followed by a projection to $H_{1}\left(\tilde{T}_{i}^{ \pm} ; \mathbb{Z}\right)$. Choose a generic pair of positive integers $r^{ \pm}$subject to

$$
r^{+}-r^{-}=-k,
$$

then there exists $\beta^{ \pm}$in $H_{2}\left(\tilde{J}^{ \pm}, \partial \tilde{J}^{ \pm} ; \mathbb{Z}\right)$ such that

$$
\partial_{0} \beta^{ \pm}=0, \text { and } \partial_{1} \beta^{ \pm}=p \cdot\left(\left[\tilde{l}^{ \pm}\right]+r^{ \pm} \cdot[\tilde{e}]\right),
$$

for some positive integer $p$, where $\tilde{l}^{ \pm}$and $\tilde{e}$ stands for corresponding elevated curves in $\tilde{T}_{1}^{ \pm}$. In fact, $\beta^{ \pm}$can be constructed dually by taking an (integral) homomorphism $H_{1}\left(\tilde{J}^{ \pm} ; \mathbb{Q}\right) \rightarrow \mathbb{Q}$ whose kernel contains the image of $H_{1}\left(\tilde{T}_{0}^{ \pm} ; \mathbb{Q}\right)$ and intersects $H_{1}\left(\tilde{T}_{1}^{ \pm} ; \mathbb{Q}\right)$ in the subspace spanned by the vector $p \cdot\left(\left[\tilde{l}^{ \pm}\right]+r^{ \pm} \cdot[\tilde{e}]\right)$. Denote by 
$\tilde{F}^{ \pm}$an elevation of $F^{ \pm}$in $\tilde{J}^{ \pm}$. Then for a positive integer $q$, there is an oriented taut subsurface $\tilde{E}^{ \pm}$of $\tilde{J}^{ \pm}$, whose relative homology class satisfies

$$
\left[\tilde{E}^{ \pm}\right]=\beta^{ \pm}+q \cdot\left[\tilde{F}^{ \pm}\right] .
$$

Moreover, choosing $q$ to be sufficiently large, $\left[\tilde{E}^{ \pm}\right]$lies in the fibered cone of the Thurston norm containing $\left[\tilde{F}^{ \pm}\right]$. This guarantees that $\tilde{E}^{ \pm}$can be arranged to be transverse to the Nielsen-Thurston suspension flow in $J^{ \pm}$, in the periodic case since $\tilde{E}^{ \pm}$is horizontal, or in the pseudo-Anosov case by Fried $[\mathrm{Fr}]$. Let $E^{ \pm}$be the properly essentially immersed subsurface in $J^{ \pm}$projected from $\tilde{E}^{ \pm}$. It follows that $E^{ \pm}$is also transverse to the Nielsen-Thurston suspension flow in $J^{ \pm}$. Moreover, there are two components $\partial E^{ \pm}$, namely, $c_{0}^{ \pm}$and $c_{1}^{ \pm}$, which are immersed in $T^{ \pm}$ projected from components of $\partial_{0} \tilde{E}^{ \pm}$and $\partial_{1} \tilde{E}^{ \pm}$, respectively. Note that $c_{0}^{+}$and $c_{0}^{-}$ represent the same immersed curve in $T$ up to homotopy, which we denote as $c_{0}$, and similarly denote $c_{1}$ for the immersed $c_{1}^{+}$and $c_{1}^{-}$.

Let $V$ be the union of $J^{+}$and $J^{-}$, glued along $T$. Glue up $E^{+}$and $E^{-}$along $c_{0}$ and $c_{1}$. The result is a partially immersed subsurface $E$ of $V$, in the sense that all boundary components of $E$ lie on a JSJ torus in the interior or on the boundary of $V$. Take two oppositely oriented copies $E$ and $\bar{E}$ of the partially immersed subsurface $E$. The components of $\partial E$ and $\partial \bar{E}$ are oppositely directed pairs of curves $\left\{b_{i}, \bar{b}_{i}\right\}$. For each pair $\left\{b_{i}, \bar{b}_{i}\right\}$, construct an essentially immersed, virtually embedded, oriented compact subsurface $R_{i}$, bounded by an equidegree finite cover of $b_{i} \sqcup \bar{b}_{i}$, locally from the side opposite to $E \sqcup \bar{E}$. This can be done by taking $R_{i}$ to be a partial PW subsurface as discussed in [DLW, Section 4]. Merge $R_{i}$ and $E \sqcup \bar{E}$ along boundary. In other words, pass to some finite cover of each subsurface and glue them up along the matched boundary components, (cf. [PW2, Subsection 3.3]). As a result, we obtain an essentially immersion of a closed oriented surface

$$
j: S \leftrightarrow M,
$$

together with an essential subsurface $E^{\prime}$ of $S$ having the following property: The restriction of $j$ to $E^{\prime}$ factorizes as a covering followed by the partial immersion:

$$
E^{\prime} \rightarrow E \rightarrow V \text {. }
$$

Observe that $\Phi(S)$ is transverse to the Nielsen-Thruston suspension flow. Indeed, over $E \sqcup \bar{E}$ this follows from our construction; over $R_{i}$ this is because a partial PW subsurface by definition does not contain geometrically infinite JSJ subsurfaces carried by hyperbolic pieces.

To see that $S$ is not aspiral, we take a directed loop $\alpha$ in $E$ which crosses $c_{0}$ and $c_{1}$ once each, assuming that $\alpha$ crosses $c_{1}$ from the - side to the + side. Let $\alpha^{\prime}$ be an elevation of $\alpha$ in $E^{\prime}$. Hence $\alpha^{\prime}$ is a loop in the almost fiber part $\Phi(S)$. From the construction, $\sigma_{M}\left(c_{0}\right)=1$ and $\sigma_{M}\left(c_{1}\right)=\left(p r^{-}+q\right) /\left(p r^{+}+q\right)$. By Lemma 7.3 and our assumption of $\theta$, the correction factor $\rho_{M}\left(\alpha^{\prime}\right)=1$. Then by Formula 7.2:

$$
s\left(\alpha^{\prime}\right)=\left(\frac{p r^{-}+q}{p r^{+}+q}\right)^{\left[\alpha^{\prime}: \alpha\right]} \neq 1 .
$$

We conclude that $\Phi(S)$ is not aspiral, so $S$ is not virtually embedded by Theorem 1.1 . 


\section{DECISION PROBLEMS}

As the last part of our discussion, we consider the decision problem for surface subgroup separability. We refer the readers to a recent survey [AFW2] of Aschenbrenner-Friedl-Wilton for background about decision problems in 3-manifold groups.

Proposition 9.1. The surface subgroups of a finitely presented closed 3-manifold group are recursively enumerable, by exhibiting a finitely presented generating set for each of them. Moreover, the separability of these subgroups can be decided.

As the Membership Problem is solvable for finitely presented 3-manifold groups [AFW2, Theorem 4.11], Proposition 9.1 implies a solution to the decision problem for the separability of a (presumed) surface subgroup in a finitely presented 3manifold group, as asserted by Corollary 1.2.

We first solve a topological decision problem.

Lemma 9.2. If $S$ is a piecewise linearly immersed triangulated closed subsurface of a closed triangulated 3-manifold $M$, then it is decidable whether or not $S$ is essentially immersed, and if yes, whether or not $S$ is virtually embedded.

Proof. We may first check if $M$ is orientable by computing its orientation character, and if $M$ is not orientable, we produce a double cover of $M$ and choose an elevation of $S$. Without loss of generality, we may assume that $M$ is orientable and $S$ is not a sphere.

Find a collection of essential spheres of $M$ which yields a Kneser-Milnor decomposition of $M$. Simplify the intersection of $S$ with the essential spheres by disk surgeries to produce an immersed closed subsurface $S^{\prime}$ in the complement. If $S^{\prime}$ is not homeomorphic to $S$ together with possibly some sphere components, then $S$ is not essentially immersed. Otherwise, replace $M$ with the closed Kneser-Milnor factor containing the non-sphere component of $S^{\prime}$. Thus we may assume that $M$ is irreducible.

Proceed to find a collection of of essential tori of $M$ which gives rise to a JSJ decomposition of $M$. If $S$ intersects a JSJ torus in a contractible loop which is homotopically nontrivial on $S$, then $S$ is not essentially immersed. Otherwise, simplify the position of $S$ by disk surgeries and discard sphere components. Further simplify the position of $S$ by annulus surgeries to obtain the induced JSJ decomposition of $S$.

Note that $S$ is essentially immersed if and only if the JSJ subsurfaces $S_{v}$ are essentially immersed in the carrier JSJ pieces $J$ of $M$. For each $S_{v}$, simutaneously run the following three processes:

- Recursively enumerate all the nontrivial elements of $\pi_{1}\left(S_{v}\right)$; at each step, decide whether the element is trivial in $\pi_{1}(J)$; return if yes.

- Recursively enumerate all the elements of $\pi_{1}(J)$; at each step, decide whether the element is a normalizer of $\pi_{1}\left(S_{v}\right)$ and not contained in $\pi_{1}\left(S_{v}\right)$; return if yes.

- Recursively enumerate all the homomorphisms from any finite index subgroups of $\pi_{1}(J)$ to $\pi_{1}\left(S_{v}\right)$; at each step, decide whether the homomorphism is a retraction to the image of $\pi_{1}\left(S_{v}\right)$; return if yes. 
One of the three processes must halt, so we have certified either that $S_{v}$ is not essentially properly immersed, or that $S_{v}$ is a virtual fiber, or that $S_{v}$ is a virtual retract.

In the first case, $S$ is not virtually immersed. In the second or the third case, $S$ is virtually immersed, and we have recognized the almost fiber part $\Phi(S)$. Moreover, we have also obtained a collection of the setup data which defines the associated $\mathbb{Q}^{\times}$-principal bundle $\mathscr{H}$ (Subsection 4.2 ) by the second process. Then we can compute the spirality character of the almost fiber part $\Phi(S)$ using Formula 4.5, and decide whether or not it always takes values in $\{ \pm 1\}$. Respectively, we can conclude whether or not $S$ is virtually embedded.

Proof of Proposition 9.1. Let $\mathcal{P}$ be a finite presentation of a closed 3-manifold group $\pi$. There is an algorithm to produce a pointed closed triangulated 3-manifold $\left(M, x_{0}\right)$ with an isomorphism $\pi_{1}\left(M, x_{0}\right) \cong \pi$, [AFW2, Lemma 3.3].

Consider any pair $(S, h)$ where $S$ is a triangulated closed surface with a base point $*$, and $h: \pi_{1}(S, *) \rightarrow \pi_{1}\left(M, x_{0}\right)$ is a homomorphism. Possibly after barycentric subdivision, we can realize $h$ by a pointed piecewise linear map $S \rightarrow M$. By Lemma 9.2, we can decide whether $S$ is essentially immersed, and if yes, whether it is virtually embedded. In other words, we can decide whether $h$ is injective, and if yes, whether the image of $h$ is separable by Przytycki-Wise [PW3]. When $h$ is injective, we return with a finitely presented generating set of the image of $h$, which is induced by the finite presentation of $\pi_{1}(S, *)$. Otherwise, we return with no output.

Recursively enumerate all the pairs $(S, h)$, then the above process outputs a complete list of all the surface subgroups of $\pi$, each given by a finitely presented generating subset, and decorated with a mark of separability.

\section{Conclusions}

In conclusion, essentially embedded closed subsurfaces in closed orientable aspherical 3-manifolds can be virtually fit into a globally interesting position, and the obstruction for essentially immersed subsurfaces to having such virtual arrangement is the spirality character of the almost fiber part. When a subsurface is locally nicely immersed with respect to a fibered cone, the spirality character can be determined more effectively.

We propose some further questions regarding suspension flow and essentially immersed subsurfaces. Let $S$ be a closed essentially immersed subsurface of a closed orientable nonpositively curved 3-manifold $M$ - the assumption on $M$ is to heuristically ensure many flexible virtual fibrations [Ag1, Ag2, L, PW2].

Question 10.1. If $S$ has no JSJ subsurface which is a vertical annulus carried by a Seifert fibered piece, does $S$ virtually satisfy the assumption of Formula 7.2?

Question 10.2. Under the assumption of Formula 7.2, do we have an effective characterization of the almost fiber part $\Phi(S)$ analogous to the finite foliation criterion of Cooper-Long-Reid [CLR1, CLR2]?

Question 10.3. How to construct a virtual fiber of $M$ with control on the degeneracy slopes? 


\section{REFERENCES}

[Ag1] I. Agol, Criteria for virtual fibering, J. Topol. 1 (2008), 269-284.

[Ag2] I. Agol, The virtual Haken conjecture, with an appendix by I. Agol, D. Groves, J. Manning, Documenta Math. 18 (2013), 1045-1087.

[AFW1] M. Aschenbrenner, S. Friedl, H. Wilton. 3-manifold groups, preprint, 149 pages, 2012. arXiv: $1205.0202 \mathrm{v} 3$.

[AFW2] _ Decision problems for 3-manifolds and their fundamental groups., preprint, 30 pages, 2014. arXiv:1405.6274v1.

[CLR1] D. Cooper, D. D. Long, A. W. Reid, Bundles and finite foliations. Invent. Math. 118 (1994), no. 1, 255-283.

[CLR2] — Finite foliations and similarity interval exchange maps. Topol. 36 (1997), no. 1, 209-227.

[CLR3] — Essential closed surfaces in bounded 3-manifolds, J. Amer. Math. Soc. (1997), $553-563$.

[DLW] P. Derbez, Y. Liu, S.-C. Wang, Chern-Simons theory, surface separability, and volumes of 3-manifolds, preprint, 43 pages, 2013. arXiv:1401.0073.

[FHS] M. Freedman, J. Hass and P. Scott, Least area incompressible surface in 3-manifolds, Invent. Math. 71 (1981), 609-642.

[Fr] D. Fried, Fibrations over $S^{1}$ with pseudo-Anosov monodromy, in: Travaux de Thurston sur les Surfaces, Astérisque, Vol. 66-67, 1979, pp. 251265.

[Ga] D. Gabai, Foliations and the topology of 3-manifolds, J. Diff. Geom. 18 (1983), no. 3, 445503.

[GO] D. Gabai, U. Oertel. Essential laminations in 3-manifolds. Ann. Math. (1989),41-73.

[HK] E. Hironaka, E. Kin, A family of pseudo-Anosov braids with small dilatation, Algebr. Geom. Topol. 6 (2006), 699-738.

[HKM] K. Honda, W. H. Kazez, G. Matić, Right-veering diffeomorphisms of compact surfaces with boundary. Invent. math. 169 (2007), no. 2, 427-449.

[JS] W. Jaco, P. B. Shalen, Seifert fibered space in 3-manifolds, Mem. Amer. Math. Soc. 21 (1979).

[Joh] K. Johannson Homotopy Equivalence of 3-Manifolds with Boundary, Lecture Notes in Math. 761, Springer-Verlag, Berlin, 1979.

[L] Y. Liu, Virtual cubulation of nonpositively curved graph manifolds, J. Topol. 6 (2013), 793822 .

[LoN] D. Long, G. Niblo, Subgroup separability and 3-manifold groups, Math. Z. 207 (1991), no. 2, 209-215.

[mM] C. T. McMullen, Polynomial invariants for fibered 3-manifolds and Teichmüller geodesics for foliations, in: Annales Scientifiques de l'École Normale Supérieure. Vol. 33. No. 4. No longer published by Elsevier, 2000.

[Ne] W. D. Neumann, Immersed and virtually embedded $\pi_{1}$-injective surfaces in graph manifold, Algebr. Geom. Topol. 1 (2001), 411-426.

[NW] G. Niblo, D. Wise, Subgroup separability, knot groups and graph manifolds, Proceedings Amer. Math. Soc. 129 (2001), 685-693.

[Ni] J. Nielsen, Surface transformation classes of algebraically finite type, Danske Vid. Selsk. Math.-Phys. Medd. 68 (1944).

[PW1] P. Przytycki, D. T. Wise, Graph manifolds with boundary are virtually special, J. Topol. 7 (2014), no. 2, 419-435.

[PW2] - Mixed 3-manifolds are virtually special, preprint, 2012, 29 pages, arXiv: 1205.6742.

[PW3] _ Separability of embedded surfaces in 3-manifolds, Compositio Mathematica 150 (2014), no. 09, 1623-1630.

[RW] J. H. Rubinstein, S.-C. Wang, $\pi_{1}$-injective surfaces in graph-manifolds, Comment. Math. Helv. 73 (1998), 499-515.

[Sc] P. Scott, Subgroups of surface groups are almost geometric, J. London Math. Soc. (2) 17 (1978), no. 3, 555-565.

[Th1] W. P. Thurston, The Geometry and Topology of Three-Manifolds, Lecture Notes, Princeton, 1977 . 
[Th2] - A norm for the homology of 3-manifolds. Mem. Amer. Math. Soc. 59 (1986), no. 339 , pp. $99-130$.

[Th2] W. P. Thurston, On the geometry and dynamics of diffeomorphisms of surfaces, Bull. Amer. Math. Soc. 19 (1988), no. 2, 417-438.

[Wi] D. Wise, The structure of groups with quasiconvex hierarchy, preprint, 2011, 186 pages, available at http://www.math.mcgill.ca/wise/papers.html.

Mathematics 253-27, California Institute of Technology, Pasadena, CA 91125

E-mail address: yliumath@caltech.edu 